IMPROVING THE RATE OF CONVERGENCE OF 'HIGH ORDER FINITE ELEMENTS' ON POLYHEDRA II: MESH REFINEMENTS AND INTERPOLATION

\author{
By \\ Constantin Bacuta \\ Victor Nistor \\ and \\ Ludmil T. Zikatanov
}

IMA Preprint Series \# 2114

(April 2006)

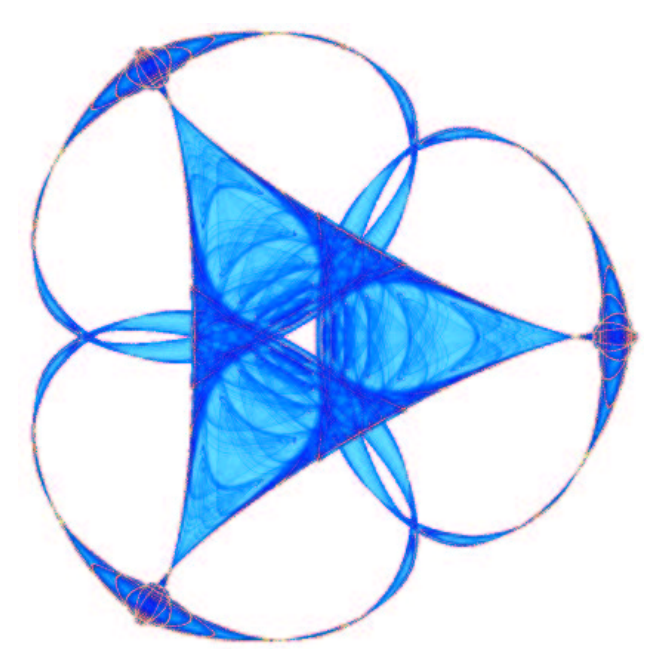

INSTITUTE FOR MATHEMATICS AND ITS APPLICATIONS

UNIVERSITY OF MINNESOTA 400 Lind Hall

207 Church Street S.E.

Minneapolis, Minnesota 55455-0436

Phone: 612/624-6066 Fax: 612/626-7370

URL: http://www.ima.umn.edu 


\title{
IMPROVING THE RATE OF CONVERGENCE OF 'HIGH ORDER FINITE ELEMENTS' ON POLYHEDRA II: MESH REFINEMENTS AND INTERPOLATION
}

\author{
CONSTANTIN BACUTA, VICTOR NISTOR, AND LUDMIL T. ZIKATANOV
}

Version: 2.1; Revised: 04/11/06; Run: April 11, 2006

\begin{abstract}
Given a bounded polyhedral domain $\Omega \subset \mathbb{R}^{3}$, we construct a sequence of tetrahedralizations (i.e., meshes) $\mathcal{T}_{k}^{\prime}$ that provides quasi-optimal rates of convergence with respect to the dimension of the aproximation space for the Poisson problem with data $f \in H^{m-1}(\Omega), m \geq 2$. More precisely, let $S_{k}$ be the Finite Element space of continuous, piecewise polynomials of degree $m \geq 2$ on $\mathcal{T}_{k}^{\prime}$ and let $u_{k} \in S_{k}$ be the finite element approximation of the solution $u$ of the Poisson problem $-\Delta u=f, u=0$ on the boundary, then $\left\|u-u_{k}\right\|_{H^{1}(\Omega)} \leq C \operatorname{dim}\left(S_{k}\right)^{-m / 3}\|f\|_{H^{m-1}(\Omega)}$, with $C$ independent of $k$ and $f$. Our method relies on the a priori estimate $\|u\|_{\mathcal{D}} \leq C\|f\|_{H^{m-1}(\Omega)}$ in certain anisotropic weighted Sobolev spaces $\mathcal{D}=\mathcal{D}_{a+1}^{m+1}(\Omega)$, with $a>0$ small and determined by $\Omega$. The weight is the distance to the set of singular boundary points (i.e., edges). The main feature of our mesh refinement is that a segment $A B$ in $\mathcal{T}_{k}^{\prime}$ will be divided into two segments $A C$ and $C B$ in $\mathcal{T}_{k+1}^{\prime}$ as follows: $|A C|=|C B|$ if $A$ and $B$ are equally singular and $|A C|=\kappa|A B|$ if $A$ is more singular than $B$. We can chose $\kappa \leq 2^{-m / a}$. This allows us to use a uniform refinement of the tetrahedra that are away from the edges to construct $\mathcal{T}_{k}^{\prime}$.
\end{abstract}

\section{Contents}

Introduction 1

1. Isotropic and anisotropic Sobolev spaces with weights 3

2. Estimates for Dirichlet's problem 10

3. Initial tetrahedralization and the general strategy 14

4. Partitioning, tetrahedralization, and refinement strategies 18

5. Interpolation and approximation on standard simplices 27

6. Interpolation on thin tetrahedra 30

7. Conclusion $\quad 34$

Appendix A. Notation 35

$\begin{array}{ll}\text { References } & 35\end{array}$

\section{INTRODUCTION}

Let $\Omega \subset \mathbb{R}^{3}$ be a bounded polyhedral domain. Consider the Poisson problem

$$
-\Delta u=f,\left.\quad u\right|_{\partial \Omega}=0
$$

The first author was partially by UDRF. L.T.Z. was supported by an NSF grant. 
where $\Delta$ is the Laplace operator. Let $S_{k} \subset H_{0}^{1}(\Omega)$ be a sequence of finite dimensional spaces of $\operatorname{dimension} \operatorname{dim}\left(S_{k}\right)$ and let $u_{k} \in S_{k}$ be the Finite Element approximation of the solution $u$ of Equation (1) for some $f \in H^{m-1}(\Omega)$. We shall say that the sequence $S_{k}$ achieves quasi-optimal rates of convergence with respect to the dimension of the space $S_{k}$ if there exists a constant $C>0$, independent of $k$ and $f$, such that

$$
\left\|u-u_{k}\right\|_{H^{1}(\Omega)} \leq C \operatorname{dim}\left(S_{k}\right)^{-m / 3}\|f\|_{H^{m-1}(\Omega)}, \quad u_{k} \in S_{k} .
$$

If $\Omega$ is bounded with smooth boundary, then it is well known $[9,12,16]$ that we can chose our sequence of tetrahedralization $\mathcal{T}_{k}^{\prime}$ to be quasi-uniform, provided that the Dirichlet boundary conditions are suitably treated. On the other hand, if $\Omega$ is not smooth, an important result of Wahlbin [31] states that a quasi-uniform sequence of triangulations will not lead to optimal rates of convergence for the sequence $u_{k}$. Nevertheless, if $\Omega$ is a polygonal domain in the plane, it was shown by Babuška in the ground breaking paper [8] that there exist sequences $S_{n}$ that will achieve optimal rates of convergence. See also Raugel [29], Apel, Sänding, and Whiteman [6], Babuška, Kellogg, and Pitkäranta [10], or the book of Oganesyan and Rukhovets [28] for another proof of this result. Yet another proof of this result was given in a previous paper of ours for polygonal domains, [14].

It is the purpose of this paper to show the existence of (and effectively construct) a sequence $S_{k}$ of finite element spaces providing quasi-optimal rates of convergence with respect to the dimension of the approximation space using the refining methods of $[10,14]$, a regularity result in the spirit of [15], and interpolation results in the spirit of [4] (see also [5]). The approximation space $S_{k}$ is associated to a sequence of conforming meshes $\mathcal{T}_{k}^{\prime}$ and consists of continuous, piecewise polynomials of degree $m$. The sequence of meshes is obtained by combining a uniform division of the tetrahedra that are far from the edges with a graded refinement toward the edges. Namely, we divide a vertex $A B$ in $\mathcal{T}_{k}^{\prime}$ to yield $A C$ and $C B$ in $\mathcal{T}_{k+1}^{\prime}$ such that, if $A$ is more singular than $B$, then $|A C| /|A B|=\kappa$, for a parameter $\kappa=2^{-m a}$. (The parameter $a$ small enough such that the regularity Theorem 2.2 is satisfied.) On the other hand, the uniform refinement procedure is such that $A B$ is divided into two equal parts if $A$ and $B$ are equally singular.

We now describe the contents of our paper. In Section 1 we recall the definitions and properties of weighted Sobolev spaces and we introduce some anisotropically weighted Sobolev spaces. We also introduce and fix an initial decomposition $\mathcal{T}_{0}$ of $\Omega$ into tetrahedra (close to the vertices), triangular prisms (close to the edges) and an interior region $\Lambda_{0}$. In Section 2, we prove a well posedness of the Poisson problem in anisotropic weighted Sobolev spaces (Theorem 2.2), generalizing the results of $[11,15,19,20,21,22,23,27]$ and others. In Section 3, we explain the general principles of our tetrahedralizations (i.e., meshes), and formulate some conditions on our tetrahedralizations that ensure quasi-optimal rates of converges (with respect to the dimension of the approximation space). In Section 4, we explain in detail the construction of our tetrahedralizations $\mathcal{T}_{n}^{\prime}$. The tetrahedralization $\mathcal{T}_{n}^{\prime}$ is obtained from a division (or partition) $\mathcal{T}_{n}$ of $\Omega$ into tetrahedra and straight triangular prisms (except $\mathcal{T}_{0}$, which is allowed also to contain an interior polyhedral region that is not a tetrahedron or a prism). Each prism in our decompositions will have a fixed diagonal ("mark") that will determine a decomposition of it into three tetrahedra, which then leads to the mesh $\mathcal{T}_{k}^{\prime}$. To obtain $\mathcal{T}_{0}$, we also have to tetrahedralize the interior region $\Lambda_{0}$ without introducing additional edges on the boundary of $\Lambda_{0}$ 
except for the marks (we allow however additional vertices and edges interior to $\left.\Lambda_{0}\right)$. In Section 5, we obtain some refinements of the usual interpolation results on standard simplices (provided that one edge is perpendicular to a face). The interpolation result of this section separates the variable $z$ from the variables $x$ and $y$ to account for different behaviors close to the edges. These interpolation results are used then in Section 6 to prove interpolation results on thin tetrahedra which finally show that our sequence of meshes $\mathcal{T}_{k}^{\prime}$ leads to quasi-optimal rates of convergence (with respect to the dimension of the approximation space) for $m \geq 2$. Our approach is summarized in Section 7, where we also state our main and final result that the sequence $S_{k}$ of continuous, order $m$ piecewise polynomials on $\mathcal{T}_{k}^{\prime}$ provide quasi-optimal rates of convergence. The main notation is recalled in the Appendix.

Acknowledgements. We thank Doug Arnold, Ivo Babuška, Jim Bramble, Hengguang Li, Anna Mazzucato, John Osborn, Lars Wahlbin, and Jinchao Xu for useful discussions. The second named author is also grateful to the ACES (Applied Computational Engineering and Sciences) Institute of the University of Texas at Austin for support while part of this work has been completed. We dedicate this paper to Ivo Babuška for his unique and foundational contributions to the development of Computational and Applied Mathematics, on the occasion of his eightieth birthday.

\section{Isotropic AND Anisotropic Sobolev SPACES With Weights}

In this section, we shall describe the various function spaces used in what follows. We begin with an initial partition of our domain, which will be used throughout the paper. We shall use the notation from our main references, $[4,5,14,11,15]$ and [23], as much as possible.

1.1. Polyhedral domains. Throughout this paper, $\Omega$ will be a fixed polyhedral domain. Let us first introduce polygonal and polyhedral domains.

Definition 1.1. A polygonal domain is a bounded, connected, open subset $D \subset \mathbb{R}^{2}$, $\partial D=\partial \bar{D}$, together with a choice of finitely many points $\left\{A_{k}\right\} \subset \partial D$ such that the boundary of $D$ is the finite union of the straight, closed segments $\left[A_{k}, A_{k}^{\prime}\right]$, called the closed sides of $D$, where $\left\{A_{k}\right\}=\left\{A_{k}^{\prime}\right\}$, each $A_{k}$ belongs to exactly two closed sides of $D$, no two distinct open sides $\left(A_{k}, A_{k}^{\prime}\right)$ have a point in common, and no point $A_{k}$ can be eliminated from this definition.

The points $A_{k}$ are called the vertices of $D$. By replacing the closed, straight segments $\left[A_{k}, A_{k}^{\prime}\right]$ with smooth curves (without self-intersections) joining $A_{k}$ to $A_{k}^{\prime}$, we obtain a curvilinear polygonal domain. We shall sometimes replace $\mathbb{R}^{2}$ with an affine space in the definition of polygonal domain.

If the boundary of $D$ is connected, then we agree that $A_{k}^{\prime}=A_{k+1}$ or $A_{k}^{\prime}=A_{1}$. However, we do not require the boundary of $D_{j}$ to be connected, in general. The condition $\partial D=\partial \bar{D}$ means that we do not allow $D$ to be on both sides of its boundary (as it is the case in domains with slits). This condition is included for simplicity and is not essential in what follows.

We are now ready to introduce polyhedral domains.

Definition 1.2. A polyhedral domain $\Omega \subset \mathbb{R}^{3}$ is a bounded, connected open set such that there exist finitely many disjoint polygonal domains $D_{j} \subset \partial \Omega=\partial \bar{\Omega}$ such that $\partial \Omega=\cup \bar{D}_{j}$, each side of any of the domains $D_{j}$ belongs to exactly one other 
domain $D_{k}$, and no adjacent faces are coplanar. The sets $D_{j}$ are called the open faces of $\Omega$, the sides of $D_{j}$ are the open edges of $\Omega$, and the vertices of $D_{j}$ are also the vertices of $\Omega$.

A note on terminology: First, the faces, edges, or domains are considered as open sets of the minimal affine space they belong to. Nevertheless, we may say "the vertex $P$ belongs to the edge $e=(A B)$ " in the sense that $P=A$ or $P=B$ (of course $A, B \in \bar{e}=[A B]$ ). However, $P \notin e$. Sometimes, to avoid confusion, we shall say "the open edge $e$ " instead of saying simply "the edge $e . "$ Similarly, when we shall say "the closed edge $e$," we shall mean $\bar{e}$. The use of "open face" and "closed face" is similar.

1.2. Initial decomposition. We begin with an initial decomposition $\bar{\Omega}=\cup_{j=0}^{N} \bar{\Lambda}_{j}$ into regions $\Lambda_{j}$ with the following properties:

(1) Each $\Lambda_{j}$ is a polyhedral domain;

(2) If $\bar{\Lambda}_{j}$ contains a vertex of $\Omega$, then it is a tetrahedron, it contains no other vertex of $\Omega$ and it intersects at most one open edge of $\Omega$;

(A region of this type will be called a type $I$ region if it does not intersect any open edge of $\Omega$ and it will be called a type $I I$ region if it intersects exactly on open edge of $\Omega$.)

(3) If $\overline{\Lambda_{j}}$ contains no vertex of $\Omega$, but intersects an open edge $e$ of $\Omega$, then $\Lambda_{j}$ is a triangular prism with basis triangles with acute angles $(i . e .,<\pi / 2)$ and with three edges parallel to $e$;

(A region of this type will be called a type III region.)

(4) The three parallel edges of our prisms are perpendicular to the bases.

(5) $\Lambda_{0}=\Omega \backslash\left(\cup_{j \geq 1} \bar{\Lambda}_{j}\right)$ and is the only region whose closure that does not intersect any closed edge of $\Omega$;

(6) We assume that our partition is conforming, in the sense that if $\bar{\Lambda}_{j}$ and $\bar{\Lambda}_{k}$ have more than a point in common, then their intersection is a common edge or a common face of both regions.

Let us make now some comments on the initial decomposition of $\Omega$. First, $\Lambda_{0}$ is the only region1 that is not a tetrahedron or a triangular prism. The regions of type I or type II are the only tetrahedra and the regions of type III are the only prisms used in our initial decomposition. Condition 4 is introduced only for convenience. One could easily adapt the proofs below to the case when the bases are parallel, but not necessarily perpendicular to the three parallel edges. With some additional work, one could consider even more general prisms, which is convenient in implementation. However, we shall leave this for later work. Other conditions can probably be relaxed as well (like Condition 2, for example, see also below).

In Section 4, we shall construct a sequence of decompositions $\mathcal{T}_{k}$ of $\Omega$ defined in part by the initial decomposition $\mathcal{T}_{0}$ and a parameter $\kappa \in(0,1 / 2]$. The decomposition $\mathcal{T}_{k}, k \geq 1$, will consist only of prisms and tetrahedra, unlike $\mathcal{T}_{0}$, which also includes a polyhedral region that is not in general a tetrahedron or a prism (the region $\left.\Lambda_{0}\right)$.

Assume $\Omega$ is the regular tetrahedron $A_{1} A_{2} A_{3} A_{4}$ represented in Figure 1.1. A picture of a possible choice of an initial decomposition of $\Omega=A_{1} A_{2} A_{3} A_{4}$ is obtained from the decomposition of Figure 1.1 by deforming the points on the edge of $\Omega$ to obtain segments perpendicular to the edge (so that Condition 4 is satified). This can be achieved for the regular tetrahedron $\Omega=A_{1} A_{2} A_{3} A_{4}$ as follows. We first 
continuously move the point $D_{1}$ on $\left[A_{1} A_{2}\right]$ such that the plane determined by $\left[D_{1} D_{13} D_{14}\right]$ is perpendicular on $\left[A_{1} A_{2}\right]$. We then similarly reposition $D_{3}$ and $D_{4}$ and the other points close to $A_{1}, A_{3}$, and $A_{4}$. These changes preserve the topology of our tetrahedralization. Let us also notice that every vertex of $\Omega$ is contained in four small tetrahedra of our initial decomposition. This is needed because no tetrahedron of our initial decomposition is allowed to intersect two open edges, as in $[4,5]$. Again, in implementation, this condition may turn out not to be necessary.

The union of the closed tetrahedra $\bar{\Lambda}_{j}$ containing a given vertex $P$ of $\Omega$ will form a neighborhood $\mathcal{V}_{P}$ of $P$ in $\bar{\Omega}$. Similarly, the union of the closed tetrahedra and the closed prisms $\bar{\Lambda}_{j}$ containing part of a given edge $e$ of $\Omega$ will form a neighborhood $\mathcal{V}_{e}$ of $e$ in $\bar{\Omega}$. These neighborhoods are analogues of the regions of influence in [15, 22], for example. In the following, by

$$
\mathcal{V}_{P}:=\cup_{P \in \bar{\Lambda}_{j}} \bar{\Lambda}_{j} \quad \text { and } \quad \mathcal{V}_{e}:=\cup_{e \cap \bar{\Lambda}_{j} \neq \emptyset} \bar{\Lambda}_{j}
$$

we shall always denote the neighborhoods introduced above.

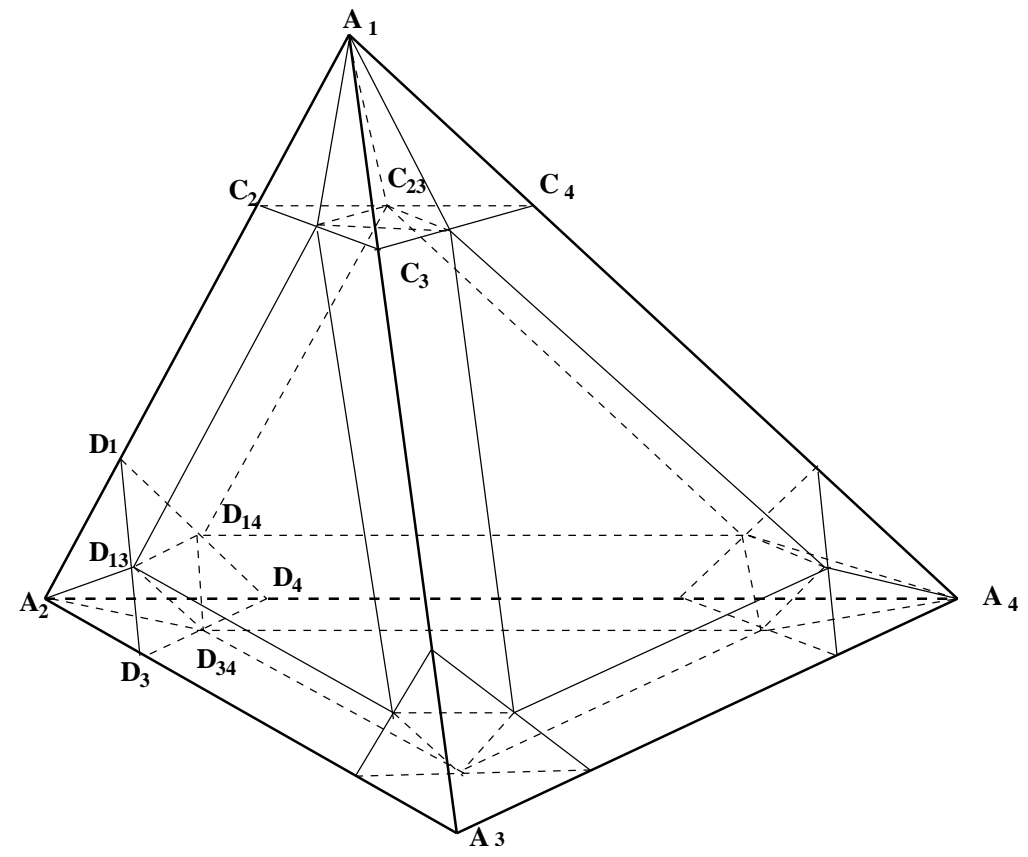

FiguRE 1.1. Initial decomposition.

1.3. Isotropic Sobolev spaces. We now introduce three classes of Sobolev spaces on $\Omega$. We shall use the standard notation for partial derivatives, namely $\partial_{j}=\frac{\partial}{\partial x_{j}}$ and $\partial^{\alpha}=\partial_{1}^{\alpha_{1}} \ldots \partial_{n}^{\alpha_{n}}$, for any multi-index $\alpha=\left(\alpha_{1}, \ldots, \alpha_{n}\right) \in \mathbb{Z}_{+}^{n}$. If $n=3$, we shall also write $\partial^{\alpha}=\partial_{x}^{\alpha_{x}} \partial_{y}^{\alpha_{y}} \partial_{z}^{\alpha_{z}}$. The usual Sobolev spaces are then

$$
H^{m}(\Omega)=\left\{u: \Omega \rightarrow \mathbb{C}, \partial^{\alpha} u \in L^{2}(\Omega),|\alpha| \leq m\right\} .
$$

(If $m$ is small, we need to consider equivalence classes of such functions.)

We now introduce two local Sobolev spaces. The space $H_{\mathrm{loc}}^{m}(\Omega)$ is the space of (equivalence classes of) functions $f: \Omega \rightarrow \mathbb{C}$ such that $f \in H^{m}(\omega)$ for every open 
subset $\omega$ satisfying $\bar{\omega} \subset \Omega$ (recall that $\Omega$ is open and bounded). Similarly, $H_{\mathrm{sl}}^{m}(\Omega)$ is the space of (equivalence classes of) functions $f: \Omega \rightarrow \mathbb{C}$ such that $f \in H^{m}(\omega)$ for every open subset $\omega$ with the property that $\bar{\omega}$ does not intersect any edge of $\Omega$.

Let us denote by $\vartheta(x)$ the distance from $x \in \Omega$ to the set of edges of $\Omega$ then the Babuška-Kondratiev spaces $\mathcal{K}_{a}^{m}(\Omega)$ are defined by

$$
\mathcal{K}_{a}^{m}(\Omega):=\left\{u, \vartheta^{|\alpha|-a} \partial^{\alpha} u \in L^{2}(\Omega), \quad|\alpha| \leq m\right\}, \quad m \in \mathbb{Z}_{+}, a \in \mathbb{R} .
$$

Then $\mathcal{K}_{a}^{m}(\Omega) \subset H_{\mathrm{sl}}^{m}(\Omega) \subset H_{\mathrm{loc}}^{m}(\Omega)$.

Recall from the previous subsection (Equation 3) the neighborhood $\mathcal{V}_{P}$ of the generic vertex $P$ (defined as the union of the closed tetrahedra $\bar{\Lambda}_{j}$ containing $P$ ) and the neighborhood $\mathcal{V}_{e}$ of the generic edge $e$ (defined as the union of the closed tetrahedra or prisms $\bar{\Lambda}_{j}$ adjacent to $e$ ). Then we shall denote by $\rho_{P}(p)$ the distance from $p \in \mathcal{V}_{P}$ to the vertex $P$. Similarly, we shall denote by $r_{e}(p)$ the distance from $p \in \mathcal{V}_{e}$ to the closed edge $e$. (This is not the distance to the line containing $e$, as in [3], for example.) Then

$$
\begin{aligned}
u \in \mathcal{K}_{a}^{m}(\Omega) \Leftrightarrow & u \in H_{\mathrm{sl}}^{m}(\Omega), \\
& \rho_{P}^{|\alpha|-a} \partial^{\alpha} u \in L^{2}\left(\mathcal{V}_{P}\right), \quad \text { and } \\
& r_{e}^{|\alpha|-a} \partial^{\alpha} u \in L^{2}\left(\mathcal{V}_{e}\right),
\end{aligned}
$$

where $|\alpha| \leq m, P$ is any vertex of $\Omega$, and $e$ is any edge of $\Omega$. (In particular, $u \in \mathcal{K}_{a}^{m}(\Omega)$ implies that $u \in H^{m}\left(\Lambda_{0}\right)$, where $\Lambda_{0}=\Omega \backslash\left(\cup_{j \geq 1} \bar{\Lambda}_{j}\right)$, as before, because the closure of $\Lambda_{0}$ does not intersect any edge of $\Omega$.)

Our Babuška-Kondratiev spaces depend on only one real parameter (excluding the smoothness index $m$ ). It is possible to define similar spaces depending on as many parameters as the total number of vertices and edges, as in [15] or in [23]. This may lead to improved regularity and well-posedness results, and hence to improved numerical methods. The theoretical treatment of the general case is, however, very similar to that considered in this paper, so we shall not consider separately this more general case.

It is elementary (and easy) to check that if $P$ is an order $m$ differential operator $P$, then

$$
P: \mathcal{K}_{a}^{s}(\Omega) \rightarrow \mathcal{K}_{a-m}^{s-m}(\Omega)
$$

is continuous for $s \geq m$. In particular, any derivative of $u \in \mathcal{K}_{a}^{s}(\Omega)$ will be in $\mathcal{K}_{a-1}^{s-1}(\Omega)$, a simple fact that will be used many times without further comment. Also, we shall need the easy fact that

$$
\mathcal{K}_{a}^{s}(\Omega) \subset \mathcal{K}_{a^{\prime}}^{s^{\prime}}(\Omega), \quad \text { for } s \geq s^{\prime} \text { and } a \geq a^{\prime},
$$

with a continuous inclusion map. The above two equations show that there is a lot of similarity in the way our Babuška-Kondratiev spaces behave with respect to the two indices $m$ and $a$.

We now recall the definition of $r_{\Omega}: \Omega \rightarrow[0, \infty)$, the smoothed distance to the edges considered in $[3,11,15]$, for example. Let $\tilde{r}_{e}(x)$ be a continuous function such that $r_{e}(x) \leq \tilde{r}_{e}(x) \leq 2 r_{e}(x)$, where $r_{e}(x)$ is the distance from $x$ to the edge $e$. We require that $\tilde{r}_{e}$ be smooth outside the edge $e$ (a property not satisfied by $r_{e}$ ) and that $r_{e}(x)=\tilde{r}_{e}(x)$ if the foot $P$ of the perpendicular from $x$ to the line containing 
$e$ is inside $e$ and that $\tilde{r}_{e}(\lambda x)=\lambda \tilde{r}_{e}(x)$ otherwise. Then we define:

$$
r_{\Omega}:=\prod_{P}\left(\rho_{P} \prod_{P \in e} \frac{\tilde{r}_{e}(x)}{\rho_{P}(x)}\right),
$$

the first product being over all vertices $P$ of $\Omega$ and the second product being over all edges $e$ of $\Omega$ containing $P$. Some of the main properties of the function $r_{\Omega}$ are that $\vartheta(x) / 2 \leq r_{\Omega}(x) \leq 2 \vartheta(x)$ and that it is smooth outside the edges.

Also, let $\rho_{\Omega}$ be a "smoothed distance to the vertices of $\Omega$ " (this is simply the distance to the origin if $\Omega=C_{\omega}$ ). In general, we chose $\rho_{\Omega}$ to be a smooth function on $\bar{\Omega}$, except maybe at the vertices, such that $\rho_{\Omega}(x)=\rho_{P}(x):=$ the distance from $x$ to $P$ if $x \in \mathcal{V}_{P}$ (i.e., if $x$ is close enough to $P$ ) and $d(x) / 2 \leq \rho_{\Omega}(x) \leq 2 d(x)$ for any $x$, where $d(x)$ is the distance from $x$ to the vertices of $\Omega$. Then we have the following lemmas that are similar to the ones in [14] and are proved as in that paper.

Lemma 1.3. We have that $r_{\Omega}^{a} \mathcal{K}_{b}^{m}(\Omega)=\mathcal{K}_{a+b}^{m}(\Omega)$ such that multiplication by $r_{\Omega}^{a}$ gives rise to an isomorphism $\mathcal{K}_{b}^{m}(\Omega) \rightarrow \mathcal{K}_{a+b}^{m}(\Omega)$. Similarly, the map $\mathcal{K}_{b}^{m}(\Omega) \ni$ $f \rightarrow \rho_{\Omega}^{a} f \in \mathcal{K}_{a+b}^{m}(\Omega)$ is continuous.

Proof. The proof is by direct calculation, see $[14,3]$ for details.

We now continue with some more properties of the Babuška-Kondratiev spaces $\mathcal{K}_{m}^{a}(\Omega)$. Let us consider the dihedral angle $D_{\alpha}:=\{0<\theta<\alpha\} \subset \mathbb{R}^{3}$. In general, a dihedral angle $D \subset \mathbb{R}^{3}$ is a set obtained from $D_{\alpha}$ by orthogonal transformations (rotations and translations). Let $\omega \subset S^{2}$ be a polygonal domain of the unit sphere $S^{2} \subset \mathbb{R}^{3}$. So all sides of $\omega$ consist of arcs of big circles of the unit sphere. Then we define $C_{\omega}:=\left\{t x^{\prime}, x^{\prime} \in \omega, t>0\right\} \subset \mathbb{R}^{3}$. A polyhedral cone is then a set obtained from a set of the form $C_{\omega}$ provided that it is not a dihedral angle. (So, in our terminology, a dihedral angle is not a polyhedral cone.)

Let $\alpha_{t}: C_{\omega} \rightarrow C_{\omega}, \alpha_{t}(x)=t x$, be the dilation by $t$. Also, we shall denote by $\alpha_{t}(f)=f \circ \alpha_{t}$ for any function defined on $C_{\omega}$. This definition is changed accordingly for polyhedral cones with the vertex not necessarily at the origin.

Lemma 1.4. Let $\Omega=C_{\omega}$. Then $\left\|\alpha_{t}(f)\right\|_{\mathcal{K}_{a}^{m}(\Omega)}=t^{a-3 / 2}\|f\|_{\mathcal{K}_{a}^{m}(\Omega)}$.

Proof. The proof is by direct calculation, as in [14].

1.4. Anisotropic Sobolev spaces. As in [4, 5, 15], we shall also need to consider certain anisotropic Sobolev spaces $\mathcal{D}_{a}^{m}(\Omega)$ that we define in this section. Our spaces seem to be slightly different from the ones defined before. We define the spaces $\mathcal{D}_{a}^{m}(\Omega), m \in \mathbb{Z}, m \geq 1, a \in \mathbb{R}$, by induction. We also define first these spaces for a dihedral angle $D_{\alpha}$, then for a polyhedral cone $C_{\omega}$, and then in general for a polyhedral domain $\Omega$.

Let $D_{\alpha}:=\{0<\theta<\alpha\}$ be the dihedral angle considered above. We let

$$
\mathcal{D}_{a}^{m}\left(D_{\alpha}\right):=\left\{u \in \mathcal{K}_{a}^{m}\left(D_{\alpha}\right), \partial_{z} u \in \mathcal{D}_{a}^{m-1}\left(D_{\alpha}\right)\right\}, \quad \mathcal{D}_{a}^{1}\left(D_{\alpha}\right):=\mathcal{K}_{1}^{1}\left(D_{\alpha}\right) .
$$

Thus the spaces $\mathcal{D}_{a}^{1}\left(D_{\alpha}\right)$ are, in fact, independent of $a$. We endow the space $\mathcal{D}_{a}^{m}\left(D_{\alpha}\right), m \geq 2$, with the norm

$$
\|u\|_{\mathcal{D}_{a}^{m}\left(D_{\alpha}\right)}^{2}:=\|u\|_{\mathcal{K}_{a}^{m}\left(D_{\alpha}\right)}^{2}+\left\|\partial_{z} u\right\|_{\mathcal{D}_{a}^{m-1}\left(D_{\alpha}\right)}^{2} .
$$

Note that if $u \in \mathcal{K}_{a}^{m}\left(D_{\alpha}\right)$, then we can only say that $\partial_{z} u \in \mathcal{K}_{a-1}^{m-1}\left(D_{\alpha}\right)$, so the condition $\partial_{z} u \in \mathcal{D}_{a}^{m-1}\left(D_{\alpha}\right)$ in the definition of the space $D_{\alpha}$ is non-trivial. The 
space $\mathcal{D}_{a}^{m}\left(D_{\alpha}\right)$ and its norm are invariant with respect to translations parallel to the $z$-axis. This definition is changed accordingly for dihedral angles not passing through the origin.

We shall need the following explicit description of the norm on the spaces $\mathcal{D}_{a}^{m}\left(D_{\alpha}\right)$. This description is similar to the one in [15], and will be used for our interpolation estimates on meshes with thin tetrahedra near the edges in Section 6 .

We shall denote by $\alpha_{\perp}=\left(\alpha_{x}, \alpha_{y}\right) \in \mathbb{Z}_{+}^{2}$ a multi-index in the first variable, and by $\partial^{\alpha_{\perp}}=\partial_{x}^{\alpha_{x}} \partial_{y}^{\alpha_{y}}$. We denote $\left|\alpha_{\perp}\right|=\alpha_{x}+\alpha_{y}$, as before. Also, we shall denote by $\mathcal{F}_{K}$ the space of functions that vanish outside the set $K$. The following lemma provides an alternative definition of the norm on the spaces $\mathcal{D}$ close to the edges.

Lemma 1.5. Let $r(p)$ be the distance from $p$ to the edge $x=y=0$ of the dihedral angle $D_{\alpha}=\{(r, \theta, z), 0<\theta<\alpha\}$ and define

$$
\begin{aligned}
& \|\| u\left\|\left.\right|_{\mathcal{D}_{a}^{m}\left(D_{\alpha}\right)} ^{2}=\sum_{k=0}^{m-2} \sum_{\left|\alpha_{\perp}\right| \leq m-k}\right\| r^{\left|\alpha_{\perp}\right|-a} \partial^{\alpha_{\perp}} \partial_{z}^{k} u \|_{L^{2}\left(D_{\alpha}\right)}^{2} \\
& +\left\|r^{-1} \partial_{z}^{m-1} u\right\|_{L^{2}\left(D_{\alpha}\right)}^{2}+\left\|\partial_{x} \partial_{z}^{m-1} u\right\|_{L^{2}\left(D_{\alpha}\right)}^{2}+\left\|\partial_{y} \partial_{z}^{m-1} u\right\|_{L^{2}\left(D_{\alpha}\right)}^{2}+\left\|\partial_{z}^{m} u\right\|_{L^{2}\left(D_{\alpha}\right)}^{2} .
\end{aligned}
$$

Let $K$ be a compact set. Then $u \rightarrow\|\| u \|_{\mathcal{D}_{a}^{m}\left(D_{\alpha}\right)}$ defines a norm on $\mathcal{D}_{a}^{m}\left(D_{\alpha}\right) \cap \mathcal{F}_{K}$ that is equivalent to the original norm $\|\cdot\|_{\mathcal{D}_{a}^{m}\left(D_{\alpha}\right)}$ on $\mathcal{D}_{a}^{m}\left(D_{\alpha}\right) \cap F_{K}$. More precisely, we have \|\|$u\|\|_{\mathcal{D}_{a}^{m}\left(D_{\alpha}\right)} \leq\|u\|_{\mathcal{D}_{a}^{m}\left(D_{\alpha}\right)} \leq C\|\| u\|\|_{\mathcal{D}_{a}^{m}\left(D_{\alpha}\right)}$.

Proof. The definition of the norm on $\mathcal{D}_{a}^{m}\left(D_{\alpha}\right)$, Equation (10) gives

$$
\begin{aligned}
\|u\|_{\mathcal{D}_{a}^{m}\left(D_{\alpha}\right)}^{2}:=\|u\|_{\mathcal{K}_{a}^{m}\left(D_{\alpha}\right)}^{2}+\left\|\partial_{z} u\right\|_{\mathcal{K}_{a}^{m-1}\left(D_{\alpha}\right)}^{2}+\ldots \\
+\left\|\partial_{z}^{m-2} u\right\|_{\mathcal{K}_{a}^{2}\left(D_{\alpha}\right)}^{2}+\left\|\partial_{z}^{m-1} u\right\|_{\mathcal{K}_{1}^{1}\left(D_{\alpha}\right)}^{2} .
\end{aligned}
$$

(Note that the last term has a slightly different form.)

The result then follows by expanding the definitions of the norms on the spaces $\mathcal{K}_{a}^{m}\left(D_{\alpha}\right), \mathcal{K}_{a}^{m-1}\left(D_{\alpha}\right), \ldots, \mathcal{K}_{a}^{2}\left(D_{\alpha}\right)$, and $\mathcal{K}_{1}^{1}\left(D_{\alpha}\right)$, Equation (4), taking into account that the distance to the edges is $\vartheta(p)=r(p)$ if $\Omega=D_{\alpha}$. To obtain the exact form given in the lemma, we also need to use that $\left\|r^{l-a} \partial^{\alpha} u\right\|_{L^{2}\left(D_{\alpha}\right)} \leq$ $C\left\|r^{k-a} \partial^{\alpha} u\right\|_{L^{2}\left(D_{\alpha}\right)}$, if $0 \leq k \leq l \leq m$, with a constant $C>0$ that is independent of $k$ and $l$.

In particular, we obtain

Corollary 1.6. Let $a \geq 0$ and $\chi: D_{\alpha} \rightarrow C$ be a smooth function. Define

$$
\|\chi\|_{m, \infty}=\sum_{k=0}^{m} \sum_{\left|\alpha_{\perp}\right| \leq m-k}\left\|r^{\left|\alpha_{\perp}\right|} \partial^{\alpha_{\perp}} \partial_{z}^{k} \chi\right\|_{L^{\infty}\left(D_{\alpha}\right)} .
$$

Then $\|\chi u\|_{\mathcal{D}_{a+1}^{m}\left(D_{\alpha}\right)} \leq C\|\chi\|_{m, \infty}\|u\|_{\mathcal{D}_{a+1}^{m}\left(D_{\alpha}\right)}$, with $C$ depending only on $m$.

Proof. It is easy to check that $\|\chi u\|_{\mathcal{K}_{a+1}^{m}\left(D_{\alpha}\right)} \leq C\|\chi\|_{m, \infty}\|u\|_{\mathcal{K}_{a+1}^{m}\left(D_{\alpha}\right)}$, if $a \geq 0$. Then our relation follows from Equation (11).

Now let $C_{\omega}=\left\{t x^{\prime}, t>0, x^{\prime} \in \omega \subset S^{2}\right\}$ be as above. We assume that $\omega$ is such that $C_{\omega}$ is not a dihedral angle, that is, we assume that $\mathcal{C}:=C_{\omega}$ is a polyhedral cone ( $\omega$ the following discussion). Let $\rho_{0}(x)$ denote the distance from $x$ to the origin $(=$ the vertex of $\mathcal{C})$. Then we let

$$
\mathcal{D}_{a}^{1}(\mathcal{C}):=\rho_{0}^{a-1} \mathcal{K}_{1}^{1}(\mathcal{C})=\left\{\rho_{0}^{a-1} v, v \in \mathcal{K}_{1}^{1}(\mathcal{C})\right\}
$$


with norm $\|u\|_{\mathcal{D}_{a}^{1}(\mathcal{C})}:=\left\|u / \rho_{0}^{a-1}\right\|_{\mathcal{K}_{a}^{1}(\mathcal{C})}$.

In general, for $m \geq 2$, let $\rho \partial_{\rho}=x \partial_{x}+y \partial_{y}+z \partial_{z}$ be the infinitesimal generator of dilations. That is $\rho \partial_{\rho}(f)=t^{-1}\left(\alpha_{t}(f)-f\right)$. Then, for $m \geq 2$, we define by induction

$$
\mathcal{D}_{a}^{m}(\mathcal{C}):=\left\{u \in \mathcal{K}_{a}^{m}(\mathcal{C}), \rho \partial_{\rho}(u) \in \mathcal{D}_{a}^{m-1}(\mathcal{C})\right\}, \quad \mathcal{C}=C_{\omega},
$$

with norm

$$
\|u\|_{\mathcal{D}_{a}^{m}(\mathcal{C})}^{2}:=\|u\|_{\mathcal{K}_{a}^{m}(\mathcal{C})}^{2}+\left\|\rho \partial_{\rho}(u)\right\|_{\mathcal{D}_{a}^{m-1}(\mathcal{C})}^{2} .
$$

We again notice that for $v \in \mathcal{K}_{a}^{m}(\mathcal{C})$, we only have $\rho \partial_{\rho} v \in \mathcal{K}_{a-1}^{m-1}(\mathcal{C})$, in general, so the condition $\rho \partial_{\rho} u \in \mathcal{D}_{a}^{m-1}(\mathcal{C})$ defining the space $\mathcal{D}_{a}^{m}(\mathcal{C})$ is non-trivial. The spaces $\mathcal{D}_{a}^{m}(\mathcal{C})$ are defined similarly for any polyhedral cone $\mathcal{C}$.

Before we define the anisotropic Sobolev spaces $\mathcal{D}_{a}^{m}(\Omega)$ in general, it is useful to discuss some properties of the various Sobolev spaces introduced so far. We begin with the following analogue of the Lemma 1.4, which will also justify in part our definition of the spaces $\mathcal{D}_{a}^{m}\left(C_{\omega}\right)$.

Lemma 1.7. Let $\Omega=C_{\omega}$. Then $\left\|\alpha_{t}(f)\right\|_{\mathcal{D}_{a}^{m}(\Omega)}=t^{a-3 / 2}\|f\|_{\mathcal{D}_{a}^{m}(\Omega)}$.

Proof. For $m=1$ this is a direct calculation. For the other values of $m$ it follows by induction, using also Lemma 1.4.

Let $C_{\omega}$ be a polyhedral cone and $e$ be an arbitrary edge of $C_{\omega}$. Let $D_{e}$ be the dihedral angle that has the edge $e$ and two faces in common with $C_{\omega}$.

Lemma 1.8. (1) Let $U \subset \bar{C}_{\omega}$ be an open set such that $\bar{U}$ does not intersect any edge of $C_{\omega}$, with the exception of $e$. Then the norms of the spaces $\mathcal{D}_{a}^{m}\left(C_{\omega}\right)$ and $\mathcal{D}_{a}^{m}\left(D_{e}\right)$ restrict to equivalent norms on $\mathcal{C}_{c}^{\infty}(U)$.

(2) Let $U \subset \bar{C}_{\omega}$ be an open set such that $\bar{U}$ does not intersect any edge of $C_{\omega}$ (no exceptions). Then the norms of the spaces $\mathcal{D}_{a}^{m}\left(C_{\omega}\right)$ and $\mathcal{D}_{a}^{m}\left(D_{e}\right)$ are equivalent to the $H^{m}(U)$ norm on $\mathcal{C}_{c}^{\infty}(U)$.

Proof. This follows from definitions.

In the above lemma, let us note that since $U$ is an open subset of $\bar{C}_{\omega}$ (instead of just $C_{\omega}$ ) it is not necessarily an open subset in $\mathbb{R}^{3}$ and, moreover, a function $\phi \in \mathcal{C}_{\mathrm{c}}^{\infty}(U)$ does not have to vanish on $\partial C_{\omega}$.

We are ready now to define the spaces $\mathcal{D}_{a}^{m}(\Omega)$ for any bounded polyhedral domain $\Omega$ by "gluing" the spaces $\mathcal{D}_{a}^{m}$ already defined using a natural partition of unity $\phi_{j}$, in which we associate one function $\phi_{j}$ to each vertex, one to each edge, and one to the interior. Recall the canonical neighborhoods $\mathcal{V}_{P}$ and $\mathcal{V}_{e}$ of a vertex $P$ or of an edge $e$ of $\Omega$ (Equation 3). For each vertex $P$, we shall denote by $C_{P}$ the polyhedral cone spanned by $P$, that is $C_{P}=\left\{t x^{\prime}, t>0, x^{\prime} \in \Omega\right\}$. Similarly, for each edge $e$, we shall denote by $D_{e}$ the dihedral angle spanned by $e$, that is, the dihedral angle that has $e$ on the edge and has two faces in common with $\Omega$.

Let $N_{e}$ and $N_{v}$ denote the total number of edges and vertices, respectively, of our bounded polyhedral domain $\Omega$. Let us chose partition of unity $\phi_{j}, j=0,1, \ldots, N:=$ $N_{e}+N_{v}$, such that

(i) $\phi_{0}=0$ in a neighborhood of the edges;

(ii) $\phi_{0}=1$ on $\Lambda_{0}$;

(iii) Assume the vertices of $\Omega$ have been denoted $P_{1}, \ldots, P_{N_{v}}$, then $\phi_{j}=1, j=$ $1, \ldots, N_{v}$, in a neighborhood the associated vertex $P_{j}$ and $\phi_{j}=0$ outside $\mathcal{V}_{P_{j}}$; 
(iv) each of the remaining functions $\phi_{j}$ (i.e., $j>N_{v}$ ) has support inside one of the sets $\mathcal{V}_{e}$, for some edge $e=e_{j}$ of $\Omega$.

We shall write $C_{j}:=C_{P_{j}}$ and $D_{j}=D_{e_{j}}$. Then $\operatorname{supp} \phi_{j} \subset C_{j}$ for $1 \leq j \leq N_{v}$ and $\operatorname{supp} \phi_{j} \subset D_{j}$ for $j>N_{v}$.

Definition 1.9. Let $\Omega$ be a bounded polyhedral domain. We define $\mathcal{D}_{a}^{m}(\Omega)$ as the space of functions $u \in \mathcal{K}_{a}^{m}(\Omega)$ such that $\phi_{j} u \in \mathcal{D}_{a}^{m}\left(C_{j}\right)$ for $1 \leq j \leq N_{v}$ and $\phi_{j} u \in \mathcal{D}_{a}^{m}\left(D_{j}\right)$ for $j>N_{v}$. We endow $\mathcal{D}_{a}^{m}(\Omega)$ with the norm

$$
\|u\|_{\mathcal{D}_{a}^{m}(\Omega)}^{2}:=\left\|\phi_{0} u\right\|_{H^{m}(\Omega)}^{2}+\sum_{j=1}^{N_{v}}\left\|\phi_{j} u\right\|_{\mathcal{D}_{a}^{m}\left(C_{j}\right)}^{2}+\sum_{j=N_{v}+1}^{N_{v}+N_{e}}\left\|\phi_{j} u\right\|_{\mathcal{D}_{a}^{m}\left(D_{j}\right)}^{2} .
$$

Lemma 1.8 guarantees that the definition of the space $\mathcal{D}_{a}^{m}(\Omega)$ is independent of the choice of the partition of unity $\phi_{j}$ with the indicated properties. In particular, since $|u|_{H^{1}(S)} \leq\|u\|_{\mathcal{K}_{a+1}^{m}(S)}$, we obtain

$$
|u|_{H^{1}(S)} \leq C_{\Omega}\|u\|_{\mathcal{D}_{a+1}^{m}(S)},
$$

where $a \geq 0, m \geq 1$, and $C_{\Omega}$ depends only on $\Omega$ and not on $S$.

If $S \subset \Omega$ is any open set, then we shall denote by $\|u\|_{S, \mathcal{D}_{a}^{m}(\Omega)}^{2}$ the quantity that we obtain if, in the integrals defining $\|u\|_{\mathcal{D}_{a}^{m}(\Omega)}^{2}$, we replace $\int_{\Omega}$ with $\int_{S}$ everywhere. In particular, for any edge $e$ and any measurable function $u: \mathcal{V}_{e} \rightarrow \mathbb{C}$, we define $\|u\|_{\mathcal{D}_{a}^{m}\left(\mathcal{V}_{e}\right)}$ by restricting the integrals defining $\|u\|_{\mathcal{D}_{a}^{m}\left(D_{e}\right)}$ to $\mathcal{V}_{e}$, where $D_{e}$ is the dihedral angle generated by $e$. Similarly, for any vertex $P$ of $\Omega$, we define $\|u\|_{\mathcal{D}_{a}^{m}\left(\mathcal{V}_{P}\right)}$ by restricting the integrals defining $\|u\|_{\mathcal{D}_{a}^{m}\left(C_{P}\right)}$ to $\mathcal{V}_{P}$, where $C_{P}$ is the polyhedral cone with vertex at $P$ generated by $\Omega$.

An equivalent norm on $\mathcal{D}_{a}^{m}(\Omega)$ is given by

$$
u \mapsto\|u\|_{H^{m}(\Omega)}^{2}+\sum_{e}\|u\|_{\mathcal{D}_{a}^{m}\left(\mathcal{V}_{e}\right)}^{2}+\sum_{P}\|u\|_{\mathcal{D}_{a}^{m}\left(\mathcal{V}_{P}\right)}^{2} .
$$

For an open subset $S \subset \Omega$, we obtain the norm

$$
\|\| u \mid\left\|_{\mathcal{D}_{a}^{m}(S)}^{2}:=\right\| u\left\|_{H^{m}(S)}^{2}+\sum_{e}\right\| u\left\|_{\mathcal{D}_{a}^{m}\left(S \cap \mathcal{V}_{e}\right)}^{2}+\sum_{P}\right\| u \|_{\mathcal{D}_{a}^{m}\left(S \cap \mathcal{V}_{P}\right)}^{2} .
$$

\section{Estimates For Dirichlet's Problem}

In this section we derive estimates on our Poisson problem (1), $-\Delta u=f$ in $\Omega$, $u=0$ on the boundary of $\Omega$, in certain anisotropic weighted Sobolev spaces. Our results are inspired by [15] and build also on a previous estimate from [11].

2.1. Preliminary results. Let us first recall the following result $\left(\mathbb{Z}_{+}=\{0,1, \ldots\}\right)$.

Theorem 2.1. Let $m \in \mathbb{Z}_{+}$and let $\Omega \subset \mathbb{R}^{3}$ be a dihedral angle, a polyhedral cone, or a bounded polyhedral domain. Then there exists $\eta>0$ such that the boundary value problem (1) has a unique solution $u \in \mathcal{K}_{a+1}^{m+1}(\Omega)$ for any $f \in \mathcal{K}_{a-1}^{m-1}(\Omega)$ and this solution depends continuously on $f$.

For $\Omega$ a bounded polyhedron and $m \geq 1$, the above result was announced in [15]. See [13] or [11] for a proof (including the case $m=0$, which is crucial in our applications). The case of the dihedral angle $D_{\alpha}:=\{0<\theta<\alpha\}$ was not treated explicitly in these papers, but can be dealt with in exactly the same way. See also $[3,7,20,21,23,26]$, and [25] for related results. See also [24] for results on mixed 
boundary value problems and an explicit treatment of the case of dihedral angles and polyhedral cones.

Since $\Delta$ maps $\mathcal{K}_{a+1}^{m+1}(\Omega) \rightarrow \mathcal{K}_{a-1}^{m-1}(\Omega)$ continuously, another way of formulating the above theorem is that the map

$$
\Delta: \mathcal{K}_{a+1}^{m+1}(\Omega) \cap\left\{\left.u\right|_{\partial \Omega}=0\right\} \rightarrow \mathcal{K}_{a-1}^{m-1}(\Omega), \quad m \in \mathbb{Z}_{+},|a|<\eta,
$$

is an isomorphism. The inverse will be denoted by $\Delta_{D, m}^{-1}$ or, simply, by $\Delta^{-1}$, when there is no danger of confusion. Let $\rho_{\Omega}$ denoted the "smoothed distance to the vertices of $\Omega$." (So $\rho=\rho_{\Omega}(p)$ is the distance to the vertices of $\Omega$ close to these vertices, and otherwise is a smooth positive function outside the vertices.) Let us notice next that the family $\rho^{-b} \Delta \rho^{b}=\Delta+2 b \rho \partial_{\rho}+\left(b^{2}+b\right)$ depends continuously on $b$ in the topology of the space of continuous maps $\mathcal{K}_{a+1}^{m+1}(\Omega) \cap\left\{\left.u\right|_{\partial \Omega}=0\right\} \rightarrow \mathcal{K}_{a-1}^{m-1}(\Omega)$. Hence we obtain that

$$
\Delta: \rho^{b} \mathcal{K}_{a+1}^{m+1}(\Omega) \cap\left\{\left.u\right|_{\partial \Omega}=0\right\} \stackrel{\simeq}{\longrightarrow} \rho^{b} \mathcal{K}_{a-1}^{m-1}(\Omega), \quad m \in \mathbb{Z}_{+},
$$

is an isomorphism for $|a|$ and $|b|$ small enough, with inverse denoted $\Delta_{m, a, b}^{-1}$.

For $|a|$ and $|b|$ small enough, we also have that $u:=\Delta_{m, a, b}^{-1} f$ is also the solution of the variational problem

$$
B(u, v):=\int_{\Omega} \nabla u \cdot \nabla v d x=\int_{\Omega} f v d x
$$

for any $v \in \rho^{-b} \mathcal{K}_{-a+1}^{1}(\Omega) \cap\left\{\left.u\right|_{\partial \Omega}=0\right\}$. This follows from the fact that $B$ also depends continuously on $a$ and $b$ and the fact that for $a=b=0$ we indeed obtain that $u$ is the solution of the variational problem (a well known, classical fact $[12,17])$. In particular, $\Delta_{0,0,0}^{-1} f=\Delta_{m, a, b}^{-1} f$ if $f \in \rho^{b} \mathcal{K}_{a-1}^{m-1}(\Omega)$, and $m \in \mathbb{Z}_{+}$, and $a$ and $b$ are small.

2.2. Anisotropic regularity. From the discussion and the results above, we shall now derive the following theorem inspired by the results in $[4,5]$, and [15] (note however that we obtain slightly more regularity than in the previous results).

Theorem 2.2. Let $m \in \mathbb{Z}_{+}$and let $\Omega \subset \mathbb{R}^{3}$ be a dihedral angle, a polyhedral cone, or a bounded polyhedral domain. Let $f \in H^{m-1}(\Omega)$ if $m \geq 1$ and $f \in \rho^{a} H^{-1}(\Omega)$ if $m=0$. We assume that $f$ has support in a fixed compact set $K$. Then there exists $\eta \in(0,1]$ such that the boundary value problem (1) has a unique solution $u \in \mathcal{D}_{a+1}^{m+1}(\Omega)$. This solution depends continuously on $f$, for any $0 \leq a<\eta$ and any $m \in \mathbb{Z}_{+}$and coincides with the solution of the variational problem (18).

Note that for $m \geq 1$, our assumptions on the data $f$ are independent of $a$. This is the case that is needed in applications. For the proof, however, we shall also need the case $m=0$. Also, it is crucial for the applications that we have in mind that $u \in \mathcal{D}_{a+1}^{m+1}(\Omega), a>0$, rather than just $u \in \mathcal{D}_{1}^{m+1}(\Omega)$. The values of $a$ for which this can be achieved $(a \in[0, \eta)$, for some $\eta>0)$, will depend, however, on the geometry of the domain $\Omega$. The results stated in [15] can give some estimates on $\eta$.

Proof. The proof of this theorem is rather long, so we shall divide it into three parts: the case of a dihedral angle, the case of polyhedral cone, and the general case. The first two cases will be proved by induction using Theorem 2.1. We shall use Equation (6) and (7) repeatedly without further comment. Also, we shall use that

$$
H_{0}^{1}(\Omega):=H^{1}(\Omega) \cap\left\{\left.u\right|_{\partial \Omega}=0\right\}=\mathcal{K}_{1}^{1}(\Omega) \cap\left\{\left.u\right|_{\partial \Omega}=0\right\}
$$


for any polyhedral domain as in the statement of our theorem (bounded or not), by the results of [11].

Step 1. $\Omega=D_{\alpha}$, a dihedral angle. We shall proceed by induction on $m$. If $m \geq 1$, we shall prove our statement under the more general assumption that $\partial_{z}^{j} f \in \mathcal{K}_{a-1}^{m-1-j}\left(D_{\alpha}\right)$ for $j \leq m-1$ and $\partial_{z}^{m} f \in \mathcal{K}_{-1}^{-1}\left(D_{\alpha}\right)$. Indeed, these assumptions are satisfied if $f$ is as in the statement of our theorem (because $a \leq 1$ and $f$ has support in a compact set.)

Let first $m=0$. Then our assumption is that $f \in H^{-1}\left(D_{\alpha}\right)$. We have

$$
H^{-1}\left(D_{\alpha}\right):=H_{0}^{1}\left(D_{\alpha}\right)^{*}=\left(\mathcal{K}_{1}^{1}\left(D_{\alpha}\right) \cap\left\{\left.u\right|_{\partial D_{\alpha}}=0\right\}\right)^{*}=: \mathcal{K}_{-1}^{-1}\left(D_{\alpha}\right) .
$$

We need to prove that the equation $-\Delta u=f,\left.u\right|_{\partial D_{\alpha}}=0$ has a unique solution $u \in$ $\mathcal{D}_{a+1}^{1}\left(D_{\alpha}\right)=\mathcal{K}_{1}^{1}\left(D_{\alpha}\right)$ and that this solution depends continuously on $f .{ }^{1}$ Indeed, in view of $H^{-1}\left(D_{\alpha}\right)=\mathcal{K}_{-1}^{-1}\left(D_{\alpha}\right)$, this follows from Theorem 2.1. This completes the proof of the initial case $(m=0)$ of our induction.

Let us consider now the induction step. Let $m \geq 1$. Then $f \in \mathcal{K}_{a-1}^{m-1}\left(D_{\alpha}\right)$. We need to show that the equation $-\Delta u=f,\left.u\right|_{\partial D_{\alpha}}=0$ (Equation (1)) has a unique solution $u \in \mathcal{D}_{a+1}^{m+1}\left(D_{\alpha}\right)$, which depends continuously on $f$. By the definition of the spaces $\mathcal{D}_{a+1}^{m+1}\left(D_{\alpha}\right)$, his means that we need to show that

(1) Equation (1) has a unique solution $u \in \mathcal{K}_{a+1}^{m+1}\left(D_{\alpha}\right)$ and that this solution satisfies $\partial_{z} u \in \mathcal{D}_{a+1}^{m}\left(D_{\alpha}\right)$.

(2) $u$ and $\partial_{z} u$ depend continuously on $f$ in the topology of the indicated spaces.

Theorem 2.1 gives that we can take $u:=\Delta_{D, m}^{-1}(f)$ and that

$$
\mathcal{K}_{a-1}^{m-1}\left(D_{\alpha}\right) \ni f \rightarrow u:=\Delta_{D, m}^{-1}(f) \in \mathcal{K}_{a+1}^{m+1}\left(D_{\alpha}\right)
$$

is continuous. We then need to show that

$$
H^{m-1}\left(D_{\alpha}\right) \ni f \rightarrow \partial_{z} u:=\partial_{z}\left(\Delta_{D, m}^{-1}(f)\right) \in \mathcal{D}_{a+1}^{m}\left(D_{\alpha}\right)
$$

is well defined (i.e., $\partial_{z} u \in \mathcal{D}_{a+1}^{m}\left(D_{\alpha}\right)$ ) and is continuous. This follows from

$$
\partial_{z} \Delta_{D, m}^{-1}(f)=\Delta_{D, m-1}^{-1}\left(\partial_{z} f\right),
$$

which we shall prove in a moment. Assume therefore Equation (20). Then $\partial_{z} f \in$ $\mathcal{K}_{a+1}^{m-2}\left(D_{\alpha}\right)$. By the induction hypothesis and the claimed relation (Equation 20), $\partial_{z} \Delta_{D, m}^{-1}(f)=\Delta_{D, m-1}^{-1}\left(\partial_{z} f\right) \in \mathcal{D}_{a+1}^{m}\left(D_{\alpha}\right)$ and depends continuously on $f$.

The remaining of this first step is to prove Equation (20). To this end, we shall use the standard techniques for proving the regularity results for boundary value problems on smooth, bounded domains [1, 2, 17, 18, 30] (in fact, what we need to prove is easier in our case). Let $\beta_{t}(x, y, z)=(x, y, z+t)$ and $\beta_{t}(u):=u \circ \beta_{t}$. Then $\beta_{t}(\vartheta)=\vartheta$ and hence the translation $\beta_{t}$ maps $\mathcal{K}_{a}^{m}\left(D_{\alpha}\right)$ to itself isometrically. Similarly, $\beta_{t}$ maps $H^{m-1}\left(D_{\alpha}\right)$ to itself continuously and we have

$$
t^{-1}\left(\beta_{t}(f)-f\right) \rightarrow \partial_{z} f \text { in } H^{m-2}\left(D_{\alpha}\right) \text { as } t \rightarrow 0 .
$$

Similarly, $u_{t}:=t^{-1}\left(\beta_{t}(u)-u\right) \in \mathcal{K}_{a+1}^{m+1}\left(D_{\alpha}\right) \subset \mathcal{K}_{1}^{1}\left(D_{\alpha}\right)$ (recall that $a \geq 0$ ). Moreover, $u_{t} \rightarrow \partial_{z} u$ in $\mathcal{K}_{a}^{m}\left(D_{\alpha}\right)$ as $t \rightarrow 0$.

$$
\Delta\left(\partial_{z} u\right)=\lim _{t \rightarrow 0} \Delta\left(u_{t}\right)=\lim _{t \rightarrow 0} f_{t}=\partial_{z} f \in H^{m-2}\left(D_{\alpha}\right) \subset \mathcal{K}_{a-1}^{m-2}\left(D_{\alpha}\right) \subset \mathcal{K}_{-1}^{-1}\left(D_{\alpha}\right),
$$

\footnotetext{
${ }^{1}$ Note that we are not claiming that there are no other solutions $u$ in other spaces, in fact, this equation has infinitely many solutions in $\mathcal{K}_{b}^{1}\left(D_{\alpha}\right)$, provided that $b$ is small enough $(b<<0)$.
} 
by the assumption that $m \geq 1$ and $a \leq 1$. Since $u=0$ on the boundary of $D_{\alpha}$ (this makes sense because $u \in \mathcal{K}_{a+1}^{m+1}\left(D_{\alpha}\right)$ is regular enough to a trace on the boundary). Since $\partial_{z} u \in \mathcal{K}_{a}^{m}\left(D_{\alpha}\right)$ we obtain that $\partial_{z}$ is also smooth enough to have a trace on the boundary and the boundary and $\partial_{z} u=0$ on the boundary of $D_{\alpha}$. Hence $\partial_{z} u=\Delta_{D, m-1}\left(\partial_{z} f\right)$. This completes the proof of the Equation (20).

Step 2. $\Omega=\mathcal{C}$, a polyhedral cone. We proceed similarly. We shall prove our statement under the more general assumptions that $f \in \rho^{a} H^{-1}(\mathcal{C})$, if $m=0$, or that $\left(\rho \partial_{\rho}\right)^{j} f \in \mathcal{K}_{a-1}^{m-1-j}(\mathcal{C})$ for $j \leq m-1$ and $\left(\rho \partial_{\rho}\right)^{m} f \in \rho^{a} \mathcal{K}_{-1}^{-1}(\mathcal{C})=\rho^{a} H^{-1}(\mathcal{C})$ for $m \geq 1$. These assumptions are satisfied if $f$ is as in the statement of our theorem because $\left(\rho \partial_{\rho}\right)^{j} f \in H^{m-1-j}(\mathcal{C})$ and $\left(\rho \partial_{\rho}\right)^{j} f$ has compact support, for $j \leq m-1$. For $j=m-1$, we also use $\left(\rho \partial_{\rho}\right)^{m-1} f=\rho\left[\partial_{\rho}\left(\rho \partial_{\rho}\right)^{m-2} f\right] \in \rho H^{-1}(\mathcal{C})$.

Let $m=0$. Then $f \in \rho^{a} H^{-1}(\mathcal{C})=\rho^{a} \mathcal{K}_{-1}^{-1}(\mathcal{C})$. Then, for $a \geq 0$ small enough, the equation $-\Delta u=f,\left.u\right|_{\partial \mathcal{C}}=0$ has a unique solution $u \in \rho^{a} H^{1}(\mathcal{C})=\rho^{a} \mathcal{K}_{1}^{1}(\mathcal{C})=$ : $\mathcal{D}_{1+a}^{1}(\mathcal{C})$, and that this solution depends continuously on $f$, by Equation (17) (which is a slight extension of Theorem 2.1). This takes care of the case $m=0$.

Let now $m \geq 1$. As in the first step, we need to show that

(1) Equation (1) has a unique solution $u \in \mathcal{K}_{a+1}^{m+1}(\mathcal{C})$ and $\rho \partial_{\rho} u \in \mathcal{D}_{a+1}^{m}(\mathcal{C})$.

(2) $u$ and $\rho \partial_{\rho} u$ depend continuously on $f$ in the topology of the corresponding spaces.

We get $u \in \mathcal{K}_{a+1}^{m+1}(\mathcal{C})$ as in the first step by using Theorem 2.1. That theorem also give that $u$ depends continuously on $f$. We then only need to show that

$$
\rho \partial_{\rho} u:=\rho \partial_{\rho}\left(\Delta_{D, m}^{-1}(f)\right) \in \mathcal{D}_{a+1}^{m}(\mathcal{C})
$$

is well defined and depends continuously on $f$ satisfying the assumptions above (i.e., $\left(\rho \partial_{\rho}\right)^{j} f \in \mathcal{K}_{a-1}^{m-1-j}(\mathcal{C})$ for $j \leq m-1$ and $\left(\rho \partial_{\rho}\right)^{m} f \in \rho^{a} \mathcal{K}_{-1}^{-1}(\mathcal{C})=\rho^{a} H^{-1}(\mathcal{C})$ ).

Let $\Delta^{\prime}$ be the Laplace operator on the unit sphere. We notice that the formula $\Delta=\rho^{-2}\left(\left(\rho \partial_{\rho}\right)^{2}+\rho \partial_{\rho}+\Delta^{\prime}\right)$ gives

$$
\Delta\left[\rho \partial_{\rho}(u)\right]=\rho \partial_{\rho}(\Delta u)+2 \Delta u=\rho \partial_{\rho}(f)+2 f .
$$

(This formula also follows from the behavior of $\Delta$ with respect to dilations $\alpha_{t}(x)=$ $t x, x \in \mathbb{R}^{3}$, as in the first step. We omit the similar details.) For $m \geq 1$ (our case), it makes sens to restrict $u$ and $\rho \partial_{\rho} u$ to the boundary, so they are both zero at the boundary. This gives

$$
\rho \partial_{\rho}(u)=\Delta_{D, m-2}^{-1}\left(\rho \partial_{\rho}(f)\right)+2 \Delta_{D, m-1}^{-1}(f) .
$$

We next show that $\Delta_{D, m-2}\left(\rho \partial_{\rho} f+2 f\right)=\Delta_{D, m-2}^{-1}\left(\rho \partial_{\rho}(f)\right)+2 \Delta_{D, m-1}^{-1}(f) \in$ $\mathcal{D}_{a+1}^{m}(\mathcal{C})$. Indeed, we have that $\mathcal{K}_{a+1}^{0}(\mathcal{C}) \subset \rho^{a} H^{-1}(\mathcal{C})$, and hence $\rho \partial_{\rho} f+2 f$ satisfies the same assumptions as $f$, but with $m$ replaced with $m-1$. The induction hypothesis then gives $u \in \mathcal{D}_{a+1}^{m+1}(\mathcal{C})$, as desired.

Step 3. $\Omega$ a bounded polyhedral domain. Let $f \in H^{m-1}(\Omega)$, if $m \geq 1$, or $f \in \rho^{a} H^{-1}(\Omega)$ if $m=0$. In any case, we have $f \in H^{-1}(\Omega)$ (because $a \geq 0$ ), and hence $u:=\Delta_{0,0,0}^{-1} f \in H_{0}^{1}(\Omega)$ is defined. (Recall that the definition of $u$ is such that $\Delta u=f$ and $u \in H_{0}^{1}(\Omega)$.)

We want to show that $u \in \mathcal{D}_{a+1}^{m+1}(\Omega)$. For $m=0$, as in the second step, this follows from the discussion preceding the statement of this theorem (which is turn based on Theorem 2.1), which shows that $u \in \rho^{a} \mathcal{K}_{1}^{1}(\Omega)=\mathcal{D}_{a+1}(\Omega)$. (Recall that 
$\rho=\rho_{\Omega}$ is now the smoothed distance to the vertices of $\Omega$.) This takes care of the case $m=0$.

Let us consider now the case $m \geq 1$. We shall proceed by induction, using the notation of Definition 1.9. In particular, $\phi_{j}$ is the partition introduced right before that definition. In particular, $\nabla \phi_{j}=0$ in a neighborhood of each vertex. Then $\Delta\left(\phi_{j} u\right)=f_{1}:=\phi_{j} f+2 \nabla \phi_{j} \cdot \nabla u+\left(\Delta \phi_{j}\right) u$.

Let us assume that $\phi_{j}$ is supported near an edge $e$. We can assume that in the neighborhood $\mathcal{V}_{e}$ of the edge $e$, all the functions $\phi_{j}$ depend only on $z$ (the coordinate along the edge). Then $\Delta\left(\phi_{j} u\right)=f_{1}:=\phi_{j} f+2 \partial_{z} \phi_{j} \partial_{z} u+\left(\partial_{z}^{2} \phi_{j}\right) u$. We have $\phi_{j} f \in$ $H^{m-1}(\Omega)$. The induction hypothesis shows that $2 \partial_{z} \phi_{j} \partial_{z} u+\left(\partial_{z}^{2} \phi_{j}\right) u \in \mathcal{D}_{a+1}^{m}(\Omega)$, and hence $f_{1}:=\phi_{j} f+2 \partial_{z} \phi_{j} \partial_{z} u+\left(\partial_{z}^{2} \phi_{j}\right) u$ satisfies the assumptions of Step 1. This shows that $\phi_{j} u \in \mathcal{D}_{a+1}^{m+1}(\Omega)$.

Let us assume now that $\phi_{j}$ is supported near a vertex $P$. Then, similarly, $f_{1}$ satisfies the assumptions of Step 2 (because $2 \nabla \phi_{j} \cdot \nabla u+\left(\Delta \phi_{j}\right) u=0$ in a neighborhood of the vertex), and hence $\phi_{j} u \in \mathcal{D}_{a+1}^{m+1}(\Omega)$. (Where $2 \nabla \phi_{j} \cdot \nabla u+$ $\left(\Delta \phi_{j}\right) u=0$ is non-zero, we use the argument of the previous paragraph.)

Finally, $\phi_{0} u$, the only remaining term of the form $\phi_{j} u$ not already considered, is supported away from the edges. Since $\Delta\left(\phi_{0} u\right)=f_{1}:=\phi_{j} f+2 \nabla \phi_{j} \cdot \nabla u+\left(\Delta \phi_{j}\right) u \in$ $H^{m-1}(\Omega)$, by induction. Elliptic regularity for smooth, bounded domains then shows that $\phi_{0} u \in H^{m+1}(\Omega) \subset \mathcal{D}_{a+1}^{m+1}(\Omega)$. Since $u=\sum \phi_{j} u$, the third step is complete and so is the proof of our theorem.

Remark 2.3. For our main result, we shall need mostly the following two ingredients: the estimates of the above theorem close to the edges and the dilation invariance of the norm on $\mathcal{D}_{a}^{m}$ for functions supported close to a vertex. The case of an infinite edge is much easier to check.

\section{INITIAL TETRAHEDRALIZATION AND THE GENERAL STRATEGY}

We now explain the general ideas and properties of our tetrahedralizations. The details of these constructions will be completed in the following sections. We begin by introducing marked prisms (i.e., triangular prisms with a choice of a diagonal on one of the faces) and explain how they are tetrahedralized. Then we explain how we obtain our initial tetrahedralization $\mathcal{T}_{0}^{\prime}$ of $\Omega$. We assume that we have fixed an initial decomposition $\mathcal{T}_{0}, \bar{\Omega}=\cup_{j} \bar{\Lambda}_{j}$, of $\Omega$ as in Subsection 1.2.

In the last two subsections we explain the properties of our sequence $\mathcal{T}_{n}^{\prime}$ of tetrahedralizations and show that they lead to quasi-optimal rates of convergence with respect to the dimension of the Finite Element space. The full details of the construction of the sequence $\mathcal{T}_{n}^{\prime}$ of tetrahedralizations will be given in the following sections.

3.1. Marked prisms and the initial tetrahedralization. In this subsection we describe the division of the prisms. Let us fix $\mathbb{P}:=A B C A^{\prime} B^{\prime} C^{\prime}$ to be a triangular prisms with $A A^{\prime}, B B^{\prime}$, and $C C^{\prime}$ parallel. Let us fix a diagonal $d$ of one of the quadrilateral faces of $\mathbb{P}$. Then we shall call $(\mathbb{P}, d)$ or $\mathbb{P}$ a marked prism and we shall call the diagonal $d$ the mark of this prism. Any marked prism $(\mathbb{P}, d)$ leads to $a$ canonical tetrahedralization of $\mathbb{P}$ after we divide the other two quadrilateral faces of $\mathbb{P}$ into two triangles using the diagonals that have a vertex in common with $d$.

For example, assume we have fixed the diagonal $B C^{\prime}$ (of the face $B C C^{\prime} B^{\prime}$ ). Then we draw the diagonals $A^{\prime} B$ and $A^{\prime} C$ (of the other two quadrilateral faces), to 
obtain a partition of $\mathbb{P}$ into the three tetrahedra $A^{\prime} A B C, A^{\prime} B C C^{\prime}$, and $A^{\prime} B^{\prime} B C^{\prime}$, as in Section 5. The mark (i.e., the fixed closed diagonal $d$ ) will always be chosen such that it does not intersect any closed edge of our polyhedral domain $\Omega$.

We shall also assume that each prism $\Lambda_{j}$ in our initial decomposition is a marked prism as follows. Let $e$ be the unique edge of $\Omega$ that intersects $\bar{\Lambda}_{j}$. We assume that the mark $d$ of $\Lambda_{j}$ (i.e., the fixed diagonal of one of the quadrilateral faces of $\Lambda_{j}$ ) belongs to the quadrilateral face of $\Lambda_{j}$ that is not adjacent $e$. In other words, all prisms in our initial decomposition are marked prisms and the mark does not intersect any edge of $\Omega$.

Two prisms in our initial decomposition $\Lambda_{j}$ and $\Lambda_{k}$ are called adjacent if they have a face in common (this implies that they correspond to the same edge $e$ of $\Omega$ ). To simplify our considerations, whenever possible, we shall chose our marks so that if $\Lambda_{j}$ and $\Lambda_{k}$ are adjacent, then the two corresponding marks (i.e., fixed diagonals) have an end point in common. In this way, only one choice has to be made for each edge of $\Omega$.

We also assume that we have fixed a tetrahedralization of $\Lambda_{0}$ such that no additional edges were introduced on the boundary of $\Lambda_{0}$ (except the marks of the prisms). We do allow however additional internal vertices, which will determine additional internal edges. Then we divide each prism into three tetrahedra as determined by the mark (this is as explained above, that is, using the fixed diagonals and such that the new diagonals of the faces adjacent to the edges have a point in common with the fixed diagonals). The resulting tetrahedralization of $\Omega$ will be called the initial tetrahedralization and our construction guarantees that this initial tetrahedralization defines a conforming mesh.

For instance, if $\Omega=A_{1} A_{2} A_{3} A_{4}$, to obtain the initial tetrahedralization, we proceed as follows. We first divide the small tetrahedra adjacent to the edges to obtain our initial decomposition, as explained in Subsection 1.2. Then we fix a diagonal of the rectangle $D_{13} D_{14} C_{23} C_{24}$ (the point $C_{23}$ is the middle of $C_{2} C_{3}$. Say we fix $D_{13} C_{24}$. This leads to a tetrahedralization of the prism $D_{1} D_{13} D_{14} C_{23} C_{24} C_{2}$ by introducing also $C_{2} D_{13}$ and $D_{1} C_{24}$ (these are the two diagonals of the faces adjacent to the edge $A_{1} A_{2}$ that have a point in common with the fixed diagonal $D_{13} C_{24}$. We proceed analogously with the other two prisms. Then we introduce the barycenter of $\Omega$ as an additional vertex and join it with all the vertices of the innermost region $\Lambda_{0}$ to obtain a tetrahedralization of this region without additional edges on its boundary (except the fixed diagonals).

3.2. The sequence of tetrahedralization. We shall construct in Section 4, for any parameter $\kappa \in(0,1 / 2]$, a sequence $\mathcal{T}_{n}$ of decompositions of $\Omega$. The decomposition $\mathcal{T}_{0}$ is our initial decomposition as in Subsection 1.2, which we assume to be fixed from now on. The decompositions $\mathcal{T}_{n}, n \geq 1$, are decompositions of $\Omega$ into finitely many tetrahedra and marked prisms, i.e., , if $T \in \mathcal{T}_{n}, n \geq 1$, then $T$ is either a tetrahedron or a marked prism $T=(\mathbb{P}, d)$, with the mark never adjacent to any edge of $\Omega$. (Note that $\mathcal{T}_{0}$ is slightly different from the other partitions because it contains also a region $\Lambda_{0}$ that is not a prism or tetrahedron.) The sequence of decompositions $\mathcal{T}_{n}$ will have the following properties. (Understanding the properties that we need will make it easier to understand the construction of our sequence of tetrahedralizations and may lead also to improved tetrahedralizations.)

(i) $\mathcal{T}_{0}$ is the initial decomposition and it satisfies the conditions of Subsection 1.2, $\mathcal{T}_{0}^{\prime}$ is the initial tetrahedralization of $\Omega$ as above (that with $\Lambda_{0}$ divided into 
tetrahedra without introducing any additional edges on the boundary of $\Lambda_{0}$, except the marks of the prisms, and the prisms divided into three tetrahedra using the mark).

(ii) $\bar{\Omega}=\cup_{T \in \mathcal{T}_{n}} \bar{T}$, that is, $\mathcal{T}_{n}$ is a decomposition of $\Omega$ into disjoint tetrahedra and straight triangular marked prisms.

(iii) $\mathcal{T}_{n}$ is a refinement of $\mathcal{T}_{0}$, in the sense that each $T \in \mathcal{T}_{n}$ is contained in exactly one $T_{0} \in \mathcal{T}_{0}$.

(iv) If we canonically divide each marked prism of $\mathcal{T}_{n}$ into three tetrahedra, then the new decomposition of $\Omega$, denoted $\mathcal{T}_{n}^{\prime}$, is a conforming mesh (that is, if two tetrahedra in $\mathcal{T}_{n}^{\prime}$ have more than an edge in common, then they have a face in common).

(v) Each prism $T \in \mathcal{T}_{n}$ has one of the three parallel edges contained in one of the edges of $\Omega$, but does not contain any vertex of $\Omega$.

(vi) Let $\mathcal{V}_{P, n}$ be the union of the closed tetrahedra of $\mathcal{T}_{n}$ that are adjacent to the vertex $P$ of $\Omega$. Then $\mathcal{V}_{P, n}$ is a neighborhood of $P$ in $\bar{\Omega}$ such that every tetrahedron $T \in \mathcal{T}_{k}, k \geq n$ that intersects $\mathcal{V}_{P, n}$ is completely contained in $\mathcal{V}_{P, n}$.

(vii) Let $\alpha_{t}$ be the dilation of ratio $t$ and center $P$. Fix a parameter $\kappa$ (usually $\left.\kappa=2^{-m / a}\right)$.Then $\alpha_{\kappa}$ maps the restriction of $\mathcal{T}_{n}$ to $\mathcal{V}_{P, k}$ to the restriction of $\mathcal{T}_{n+1}$ to $\mathcal{V}_{P, k+1}$.

(viii) The number $k_{n, j}$ of regions $T \in \mathcal{T}_{n}$ that are contained in either of the sets $\Lambda_{j}$ of the initial decomposition satisfies $C^{-1} 2^{3 n} \leq k_{n, j} \leq C 2^{3 n}$, with $C$ independent of $n$ and $j$.

(ix) Let $S_{n}$ be the space of continuous, piecewise polynomials of degree $m$ on $\mathcal{T}_{n}^{\prime}$. Let $X=\Omega \backslash \mathcal{V}_{P, 1}$ and $u \rightarrow u_{I, n}$ be the Lagrange interpolant associated to the " $m$-simplex" and the mesh $\mathcal{T}_{n}^{\prime}$ restricted to $X$. If $\kappa \leq 2^{-m / a}$, there exists a constant $C>0$ such that

$$
\left|u-u_{I, n}\right|_{H^{1}(X)} \leq C 2^{-n m}\|u\|_{\mathcal{D}_{a+1}^{m+1}(X)}
$$

for any $u \in \mathcal{D}_{a+1}^{m+1}(\Omega), u=0$ on the boundary.

From now on, $S_{n}$ be denote the Finite Element spaces of continuous, piecewise polynomial of order $m$ on the mesh $\mathcal{T}_{n}^{\prime}$ with Dirichlet boundary conditions.

Let us now make the simple, but important, remark that Condition (viii) guarantees that

$$
C^{-1} 2^{3 n} \leq \operatorname{dim} S_{n} \leq C 2^{3 n},
$$

where $C$ depends on $m$ and $\Omega$, but not on $n$.

Remark 3.1. Condition ix will be shown to be true for $m \geq 2$. The results of [4] (see also the references therein) indicate that this condition is not true for $m=1$. For $m=1$, it is necessary to use an "averaged interpolant," as in [4], but we shall not address this issue in this paper.

3.3. Quasi-optimal rates of convergence. Let us denote by $\mathcal{T}_{n}^{\prime}$ the associated tetrahedralization of $\Omega$, as before. Let $S_{n}$ be the Finite Element space of continuous, piecewise polynomials on $\mathcal{T}_{n}^{\prime}$, as we have agreed. Also, let us denote by $u_{I, n}$ the Lagrange interpolant of $u$ associated to the $m$-simplex (i.e., to uniformly distributed nodes). Assume that we have constructed a sequence $\mathcal{T}_{n}$ of decompositions of $\Omega$ satisfying the Conditions (i-ix) of the previous subsection. (So the results below 
will be proved in this paper to be true only under the additional assumption $m \geq 2$; they will probably remain true for $m=1$ with a different choice of the interpolant, see [4] and Remark 3.1.)

Theorem 3.2. Assume that Conditions $(i-i x)$ are satisfied. Let $a \in(0,1]$ and $0<\kappa \leq 2^{-m / a}$. Then there exists $C>0$ such that

$$
\left|u-u_{I, k}\right|_{H^{1}(\Omega)} \leq C 2^{-k m}\|u\|_{\mathcal{D}_{a+1}^{m+1}(\Omega)},
$$

for any $u \in \mathcal{D}_{a+1}^{m+1}(\Omega), u=0$ on the boundary, and any $k \in \mathbb{Z}_{+}$.

Proof. Let us fix $P$ and let $Y_{n}:=\mathcal{V}_{P, n} \backslash \mathcal{V}_{P, n+1}$. Then $Y_{0}:=\mathcal{V}_{P, 0} \backslash \mathcal{V}_{P, 1} \subset X:=$ $\Omega \backslash \cup_{Q} \mathcal{V}_{Q, 1}$. By Condition (vi), $\mathcal{T}_{k}, k>n$, provides a decomposition of $Y_{n}$ ( $Y_{n}$ will be contained in the union of the closures of the regions of $\mathcal{T}_{k}$ that intersect $Y_{n}$ ). In particular, $Y_{n}$ is tetrahedralized with tetrahedra in $\mathcal{T}_{k}^{\prime}$, for $k>n$.

Let $u \in \mathcal{D}_{a+1}^{m+1}(\Omega)$. We claim that we can find a constant $C$ independent of $n$ and $k$ such that

$$
\begin{gathered}
\left|u-u_{I, k}\right|_{H^{1}\left(Y_{n}\right)} \leq C 2^{-k m}\|u\|_{\mathcal{D}_{a+1}^{m+1}\left(Y_{n}\right)}, \quad 1 \leq n<k, \quad \text { and } \\
\left|u-u_{I, k}\right|_{H^{1}\left(\mathcal{V}_{P, k}\right)} \leq C 2^{-k m}\|u\|_{\mathcal{D}_{a+1}^{m+1}\left(\mathcal{V}_{P, k}\right)} .
\end{gathered}
$$

This is enough to prove our result, since the decomposition of $\Omega$ into the regions $\mathcal{V}_{P, k}, Y_{n}, 1 \leq n \leq k-1$, and $X:=\Omega \backslash \cup_{P} \mathcal{V}_{P, 1}$, together with Equation (23) and Condition (ix) (with $n$ replaced with $k$ ) give

$$
\begin{array}{r}
\left|u-u_{I, k}\right|_{H^{1}(\Omega)}^{2}=\sum_{P}\left(\left|u-u_{I, k}\right|_{H^{1}\left(\mathcal{V}_{P, k}\right)}^{2}+\sum_{n=1}^{k-1}\left|u-u_{I, k}\right|_{H^{1}\left(Y_{n}\right)}\right)+\left|u-u_{I, k}\right|_{H^{1}(X)}^{2} \\
\leq C 2^{-2 k m} \sum_{P}\left(\|u\|_{\mathcal{D}_{a+1}^{m+1}\left(\mathcal{V}_{P, k}\right)}^{2}+\sum_{n=1}^{k-1}\|u\|_{\mathcal{D}_{a+1}^{m+1}\left(Y_{n}\right)}^{2}\right)+C 2^{-2 k m}\|u\|_{\mathcal{D}_{a+1}^{m+1}(X)}^{2} \\
=C 2^{-2 k m}\|u\|_{\mathcal{D}_{a+1}^{m+1}(\Omega)}^{2}
\end{array}
$$

with a constant $C>0$ independent of $k$ and $n$.

To prove our Claim, Equation (23), let $v \in \mathcal{D}_{a+1}^{m+1}\left(Y_{n}\right)$. Let $t=\kappa^{n} \leq 2^{-n m / a}$ and $\alpha_{t}(x):=t x$. Then $\alpha_{t}$ maps $Y_{0}$ to $Y_{n}$. Let $v=u \circ \alpha_{t}$, which will then be a function defined on $Y_{0}$. Condition (vii) and the behaviour of the Lagrange interpolant under change of coordinates give $u_{I, k} \circ \alpha_{t}=v_{I, k-n}, k>n$. In turn, Lemma 1.7 and Condition (ix) give, for $t=\kappa^{n} \leq 2^{-n m / a}$,

$$
\begin{aligned}
\left|u-u_{I, k}\right|_{H^{1}\left(Y_{n}\right)}=t^{1 / 2} & \left|u \circ \alpha_{t}-u_{I, k} \circ \alpha_{t}\right|_{H^{1}\left(Y_{0}\right)}=t^{1 / 2}\left|v-v_{I, k-n}\right|_{H^{1}\left(Y_{0}\right)} \\
& \leq C_{0} t^{1 / 2} 2^{-(k-n) m} q\|v\|_{\mathcal{D}_{a+1}^{m+1}\left(Y_{0}\right)} \leq C_{0} 2^{-k m}\|u\|_{\mathcal{D}_{a+1}^{m+1}\left(Y_{n}\right)} .
\end{aligned}
$$

This proves the first part of Equation (23).

To prove the second part of Equation (23), let $u \in H^{1}\left(\mathcal{V}_{P, k}\right)$. Also, let $t=\kappa^{k} \leq$ $2^{-k m / a}, \alpha_{t}(x)=t x$, as before, and $v=u \circ \alpha_{t}$. Then $\alpha_{t}$ maps $\mathcal{V}_{P, 0}$ to $\mathcal{V}_{P, k}$ and hence $v$ will be defined on $\mathcal{V}_{P, 0}$. We shall again use the dilation $\alpha_{t}$ to define $v=u \circ \alpha_{t}$, which will then be a function defined on $\mathcal{V}_{P, 0}$. Condition (vii) and the behaviour of the Lagrange interpolant under change of coordinates give $u_{I, k} \circ \alpha_{t}=v_{I, 0}$. Let $\chi: \bar{\Omega} \rightarrow[0, \infty)$ be a smooth function that is equal to 0 in a neighborhood of the edges, but is equal to 1 at all other nodal points of $\mathcal{T}_{0}^{\prime}$. Then $v_{I, 0}=(\chi v)_{I, 0}$ on 
$\mathcal{V}_{P}=\mathcal{V}_{P, 0}$, because $u=0$ at the boundary. In turn, Lemma 1.7 and a standard interpolation estimate give, for $t=\kappa^{k} \leq 2^{-k m / a}$,

$$
\begin{aligned}
& \quad t^{-1 / 2}\left|u-u_{I, k}\right|_{H^{1}\left(\mathcal{V}_{P, k}\right)}=\left|u \circ \alpha_{t}-u_{I, k} \circ \alpha_{t}\right|_{H^{1}\left(\mathcal{V}_{P, 0}\right)}=\left|v-v_{I, 0}\right|_{H^{1}\left(\mathcal{V}_{P, 0}\right)} \\
& \leq\left(|v-\chi v|_{H^{1}\left(\mathcal{V}_{P, 0}\right)}+\left|\chi v-(\chi v)_{I, 0}\right|_{H^{1}\left(\mathcal{V}_{P, 0}\right)}\right) \leq C\left(|v|_{H^{1}\left(\mathcal{V}_{P, 0}\right)}+|\chi v|_{H^{m+1}\left(\mathcal{V}_{P, 0}\right)}\right) \\
& \leq C\left\|u \circ \alpha_{t}\right\|_{\mathcal{D}_{a+1}^{m+1}\left(\mathcal{V}_{P, 0}\right)}=C t^{a-1 / 2}\|u\|_{\mathcal{D}_{a+1}^{m+1}\left(\mathcal{V}_{P, k}\right)} \leq C t^{-1 / 2} 2^{-k m}\|u\|_{\mathcal{D}_{a+1}^{m+1}\left(\mathcal{V}_{P, k}\right)} .
\end{aligned}
$$

This completes the proof of our claim (Equation 23) and hence the proof of our theorem.

Let $f \in L^{2}(\Omega)$ and $u \in H^{1}(\Omega)$ be the weak solution of

$$
-\Delta u=f \text { in } \Omega, u=0 \text { on } \partial \Omega \text {. }
$$

Let $u_{n} \in S_{n}$ be the discrete solution of this equation, that is, the solution of the equation $B\left(u_{n}, v_{n}\right)=\int_{\Omega} f v_{n}(x) d x$, for all $v_{n} \in S_{n}$. Then $\left|u-u_{n}\right|_{H^{1}(\Omega)}$ will be called the finite element error. A standard application of Cea's Lemma [12, 16] and of Theorems 2.2 and 3.2 give then the following theorem.

Theorem 3.3. Let $S_{n}$ be the Finite Element spaces of continuous piecewise polynomials of degree $m$ associated to a tetrahedralization $\mathcal{T}_{n}^{\prime}$ of $\Omega$. Assume as above that $\mathcal{T}_{n}^{\prime}$ is associated to a sequence of decompositions $\mathcal{T}_{n}$ satisfying the conditions (i-ix) of Subsection 3.2. Let $a>0$ be as in Theorem 2.2 and $\kappa \leq 2^{-m / a}$. Then there exists $C>0$ such that

$$
\left|u-u_{n}\right|_{H^{1}(\Omega)} \leq C 2^{-n m}\|u\|_{\mathcal{D}_{a+1}^{m+1}} \leq C \operatorname{dim}\left(S_{n}\right)^{-m / 3}\|f\|_{H^{m-1}(\Omega)},
$$

for a constant $C$ independent of $n$ and $f \in H^{m-1}(\Omega)$.

Note that the assumptions of the above theorem imply that $\operatorname{dim}\left(S_{n}\right) \leq C 2^{3 n}$ and $\|u\|_{\mathcal{D}_{a+1}^{m+1}} \leq C_{a}\|f\|_{H^{m-1}(\Omega)}$. Also, let us notice that we make no additional assumption on the regularity of $u$.

Since for $f \in H^{m-1}(\Omega)$ we cannot expect anything better than $u \in H_{\mathrm{loc}}^{m+1}(\Omega)$ and $\operatorname{dim}\left(S_{n}\right) \sim 2^{3 n}$, if follows that the order of the error $\left|u-u_{n}\right|_{H^{1}(\Omega)}$ is of the same order of magnitude as the interpolation error $\inf _{\chi \in S_{n}}|u-\chi|_{H^{1}(\Omega)}$. In this sense, the rate of convergence provided by Theorem 3.3 is quasi-optimal.

The existence of a sequence $\mathcal{T}_{n}^{\prime}$ satisfying the conditions of Theorem 3.3 for $m \geq 2$ will be proved in the following sections. Our procedure will likely have to be slightly changed if $m=1$, by replacing the Lagrange interpolant with an averaged interpolant, as in [4]. The details of this still need to be worked out. See also Remark 3.1.

\section{Partitioning, tetrahedralization, And Refinement strategies}

We now present a procedure that will associate to a bounded, polyhedral domain $\Omega$, an initial decomposition $\mathcal{T}_{0}$ of $\Omega$ into tetrahedra and marked prisma, an initial tetrahedralization as in Subsection 3.1 (of the preceding section), and a parameter $\kappa \in(0,1 / 2]$, a sequence $\mathcal{T}_{n}$ of decompositions of $\Omega$ into finitely many tetrahedra and marked prisms satisfying the conditions of Subsection 3.2. This construction will involve no further choices, so it is canonical (i.e., algorithmic). The verification of Condition (ix), however, will only be completed in Section 6 . 
The tetrahedralization $\mathcal{T}_{n}^{\prime}$ of $\Omega$, for any $n$, is obtained from the decomposition $\mathcal{T}_{n}$ of $\Omega$ by dividing each marked prism into three tetrahedra, as determined by the mark and explained in Subsection 3.1.

4.1. The parameter $\kappa$ and the refinement of edges and faces. Our meshes, tetrahedralizations, and partitions of $\Omega$ will depend on the initial decomposition, the initial tetrahedralization and a parameter $\kappa \in(0,1 / 2]$. For $\kappa=1 / 2$ we shall obtain a quasi-uniform sequence of meshes. To obtain quasi-optimal rates of convergence with respect to the dimension of the Finite Element Space of order $m$ piecewise polynomials, we usually need to take $\kappa$ small. For example, if $0<a<\eta$, where $\eta$ is as in Theorem 2.2, we shall prove that we recover quasi-optimal rates of convergence (with respect to the dimension of the FEM space of order $m$ piecewise polynomials) for

$$
\kappa=2^{-m / a} .
$$

See Theorem 3.3. This is the same choice of the parameter $\kappa$ as in [14], where it was seen to be optimal for $m=1$ and a re-entrant corner in numerical tests in [14]. (This remains to be establish in three dimensions.)

Given a point $P \in \bar{\Omega}$, we shall say that $P$ is of type $\mathbf{V}$ if it is a vertex of $\Omega$; we shall say that $P$ is of type $\mathbf{E}$ if it is on an open edge of $\Omega$. Otherwise, we shall say that it is of type $\mathbf{S}$. Note that the type of a point depends only on $\Omega$ and not on any partition or meshing. Then the initial tetrahedralization will consist of edges of type $\mathbf{V E}, \mathbf{V S}, \mathbf{E S}, \mathbf{E E}:=\mathbf{E}^{2}$, and $\mathbf{S}^{2}$. (The way our initial decomposition and initial tetrahedralization was defined, no edge will be of type $\mathbf{V V}$.) The points of type $\mathbf{V}$ will be regarded as more singular than the points of type $\mathbf{E}$, and the points of type $\mathbf{E}$ will be regarded as more singular than the points of type $\mathbf{S}$.

Let $A B$ be an edge in one of our decompositions $\mathcal{T}_{n}$. Then in $\mathcal{T}_{n+1}$, this edge will be decomposed in two segments, $A C$ and $C A$, such that $|A C|=\kappa|A B|$ if $A$ is more singular than $B$ (i.e., if $A B$ is of type $\mathbf{V E}, \mathbf{V S}$, or $\mathbf{E S}$ ). In particular, $C$ will be closer to the more singular point (except when $\kappa=1 / 2$ ). If $A$ and $B$ are as singular (i.e., if $A B$ is of type $\mathbf{E}^{2}$ or $\mathbf{S}^{2}$ ), then we take $C$ to be the middle of $A B$. See Figure 4.1.

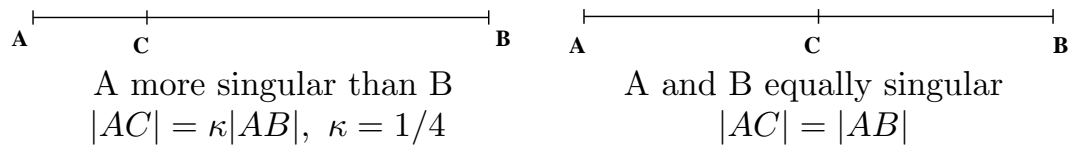

Figure 4.1. Edge decomposition

Let $A B C$ be a triangle in the decomposition $\mathcal{T}_{n}$. Then in $\mathcal{T}_{n+1}$, this triangle will be divided into four other triangles, with the exception when $A B C$ is of type VES. This will be achieved as follows. We divide each side of $A B C$ into two segments, as explained above. Then we divide $A B C$ into four triangles by joining the three new points. (For triangles of type ESS, this is discussed again in Subsection 6.1.)

On the other hand, if $A B C$ is of type VES (with $B$ of type $\mathbf{E}$ ), then we remove the newly introduced segment that is opposite $B$. This will divide $A B C$ into two triangles and a quadrilateral, with $B$ belonging to this quadrilateral and not adjoint to any of the two triangles. The newly formed quadrilateral will belong to a prism in $\mathcal{T}_{n+1}$. 


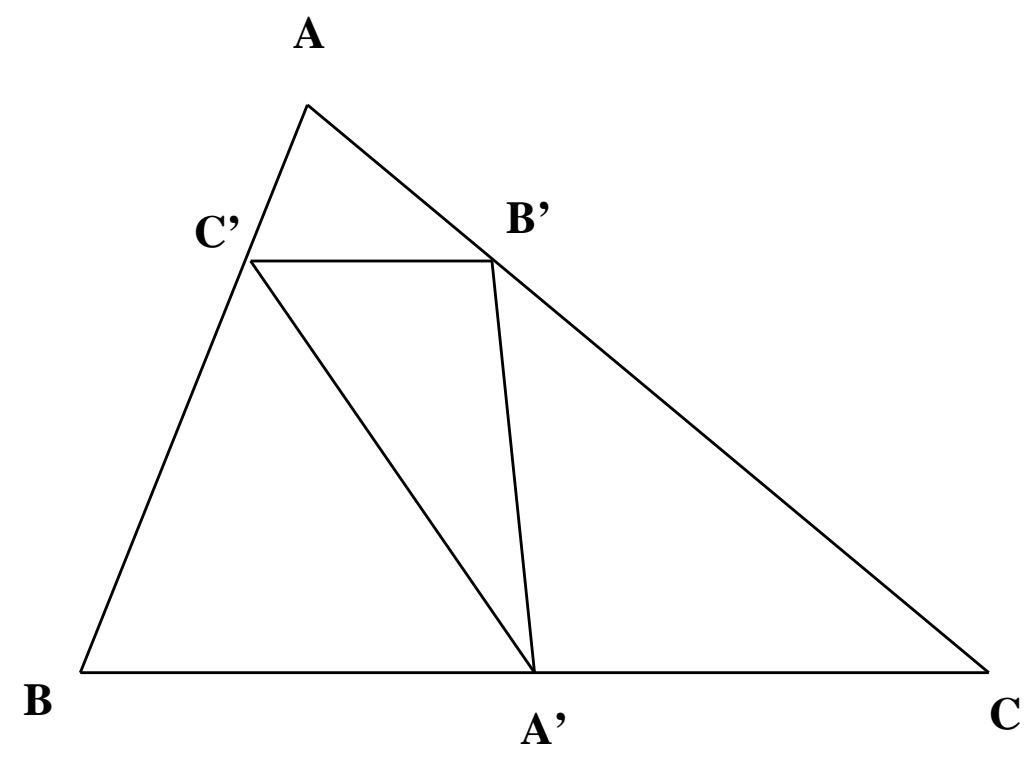

Figure 4.2. Face decomposition: $A$ of type $\mathbf{V}$ or $\mathbf{E}, B$ and $C$ of type $\mathbf{S},\left|A C^{\prime}\right|=\kappa|A B|,\left|A B^{\prime}\right|=\kappa|A C|,\left|A^{\prime} B\right|=\left|A^{\prime} C\right|, \kappa=1 / 4$

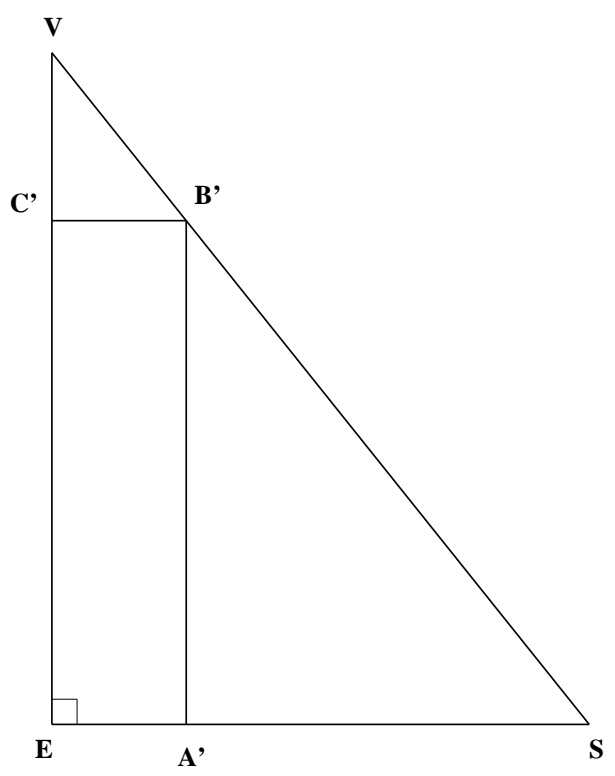

Figure 4.3. VES decomposition: $\left|V C^{\prime}\right|=\kappa|V E|, \quad\left|V B^{\prime}\right|=$ $\kappa|V S|,\left|E A^{\prime}\right|=\kappa|E S|, A^{\prime} C^{\prime}$ was removed, $\angle E=90^{\circ}$

Let $A B C D$ be a quadrilateral that appears in a prism of the decomposition $\mathcal{T}_{n}$. Then in $\mathcal{T}_{n+1}$, this quadrilateral is divided into four quadrilaterals by joining the two additional points on the opposite sides of $A B C D$ (these additional points were obtained as explained above). To obtain $\mathcal{T}_{n}^{\prime}$, we must further divide each quadrilateral into two triangles using one of the diagonals. The choice of this 
diagonal is that it is either the mark of one of the prisms or that it has a point in common with a mark of one of the prisms. See Figure 4.8.

To summarize, each edge, triangle, or quadrilateral that appears in a tetrahedron or prism in the decomposition $\mathcal{T}_{n}$ is divided in the decomposition $\mathcal{T}_{n+1}$ in an intrinsic way, which depends only on the type of the vertices (of that edge, triangle, or quadrilateral) and nothing else. In particular, the way that a face in $\mathcal{T}_{n}$ is divided to yield $\mathcal{T}_{n+1}$ does not depend on the type of the other vertices of the tetrahedron or prism to which it belongs. This ensures that the tetrahedralization $\mathcal{T}_{n+1}^{\prime}$, which is obtained from $\mathcal{T}_{n+1}$ by dividing each prism in three tetrahedra, is a conforming mesh.

4.2. Division of tetrahedra and prisms. Unlike the edges and faces that are part of $\mathcal{T}_{n+1}$, the tetrahedra that are part of $\mathcal{T}_{n+1}$ are usually not obtained by dividing a region in $\mathcal{T}_{n}$. In the following three sections we shall describe in detail how the regions of $\mathcal{T}_{n}$ are obtained by using three refining procedures:

(i) The non-uniform refinement is applied to a tetrahedron of type VESS or $\mathbf{V S}^{3}$ in $\mathcal{T}_{n}$ to yield regions of $\mathcal{T}_{n+1}$ that will be either prisms or tetrahedra of various types.

(ii) The uniform refinement of level $k$ is applied to the tetrahedron of type $\mathbf{S}^{4}$ that is either a tetrahedron in the initial tetrahedralization or is obtained by non-uniform refinement. When the uniform refinement of level $k$ is applied to the tetrahedron of type $\mathbf{S}^{4}$ of $\mathcal{T}_{n}^{\prime}$, it leads to tetrahedra of the decomposition $\mathcal{T}_{n+k}$ (which are also tetrahedra of type $\mathbf{S}^{4}$ of the tetrahedralization $\mathcal{T}_{n+k}^{\prime}$ ).

(iii) The semi-uniform refinement of level $k$ is applied to prisms that are either part of the initial decomposition $\mathcal{T}_{0}$ or are part of the decomposition $\mathcal{T}_{n}$ as a result of a non-uniform division of a tetrahedron of $\mathcal{T}_{n-1}$. When the semiuniform refinement of level $k$ is applied to a marked prism in $\mathcal{T}_{n}$, it leads to $2^{3 k}$ marked prisms of the decomposition $\mathcal{T}_{n+k}$.

It will follow by induction that every tetrahedron in the decompositions $\mathcal{T}_{k}$, $k \geq 0$, is of the type VESS, $\mathbf{V S}^{3}$, or $\mathbf{S}^{4}$, so we need not consider any other type of tetrahedron.

The general method for obtaining $\mathcal{T}_{n}$ and $\mathcal{T}_{n}^{\prime}$ is as follows. First, recall that the tetrahedralization $\mathcal{T}_{n}^{\prime}$ is obtained from $\mathcal{T}_{n}$ by dividing each prism in $\mathcal{T}_{n}$ in three tetrahedra by using its mark. On the other hand, $\mathcal{T}_{n}$ is not obtained inductively from $\mathcal{T}_{n-1}$, but is rather obtained from the initial decomposition $\mathcal{T}_{0}$ and the initial tetrahedralization $\mathcal{T}_{0}^{\prime}$. Assume $\mathcal{T}_{k}, 0 \leq k<n$ were defined. We then define $\mathcal{T}_{n}$ by dividing certain regions of $\mathcal{T}_{k}, 0 \leq k<n$ as follows:

(i) If $\Lambda$ is a tetrahedron of type $\mathbf{S}^{4}$ in the initial tetrahedralisation $\mathcal{T}_{0}^{\prime}$, then we apply to $\Lambda$ the level $n$ of uniform refinement (in this case $\Lambda \subset \Lambda_{0}$ );

(ii) If $\Lambda$ is a marked prism of the initial decomposition $\mathcal{T}_{0}$, the we apply to $\Lambda$ the semi-uniform refinement of level $n$ (in this case, $\Lambda \cap \Lambda_{0}=\emptyset$ );

(iii) If $\Lambda$ is a tetrahedron of type $\mathbf{S}^{4}$ obtained by applying the non-uniform refinement procedure to some tetrahedron $T$ in some $\mathcal{T}_{k}, 0 \leq k<n$, then we apply to $\Lambda$ the uniform refinement of level $n-k$ of (in this case, $T$ must be of type VESS or $\mathbf{V S}^{3}$ ).

(iv) If $\Lambda$ is a marked prism obtained by applying the non-uniform refinement procedure to some tetrahedron $T$ in some $\mathcal{T}_{k}, 0 \leq k<n$, then we apply 
to $\Lambda$ the level $n-k$ of semi-uniform refinement (again, $T$ must be of type VESS or $\mathbf{V S}^{3}$ ).

By a performin the level 0 of a refinement to a region we mean that we do not change that region. Then $\mathcal{T}_{n}$ is consists of the regions defined above and the tetrahedra of type VESS or $\mathbf{V S}^{3}$ obtained by the non-uniform refinement of a tetrahedron of the same type in $\mathcal{T}_{n-1}$.

All the regions considered in (i)-(iv) above and all the resulting regions that define $\mathcal{T}_{n}$ are disjoint.

4.3. Uniform refinement. In this subsection, $T \in \mathcal{T}_{k}^{\prime}$ will be a tetrahedron of type $\mathbf{S}^{4}$ obtained in one of the following way:

(i) $T$ is a tetrahedron $T \subset \Lambda_{0}$ of the initial tetrahedralization $\mathcal{T}_{0}^{\prime}$ or

(ii) $T$ is a tetrahedron of type $\mathbf{S}^{4}$ obtained by a non-uniform division of a tetrahedron of type $\mathbf{V S}^{3}$ or $\mathbf{V E S} \mathbf{E}^{2}$.

We now describe a uniform refinement strategy for dividing $T$. To obtain $\mathcal{T}_{n}^{\prime}$, we need to apply to our tetrahedron $T \in \mathcal{T}_{k}^{\prime}$ the level $n-k$ uniform refinement. We stress that this is not an inductive procedure, that is, if we apply to $T$ a the level $j$ of uniform refinement and then to each of the resulting tetrahedra we apply the level $i$ of uniform refinement, we do not obtain the level $i+j$ of uniform refinement of $T$. It is therefore necessary to keep track of how $T$ has first appeared.

Let $T=A_{1} A_{2} A_{3} A_{4}$ be the given tetrahedron and let $A_{i j}$ denote the midpoints of the edges $A_{i} A_{j},(i<j)$. The edges of the octahedron

$$
\mathcal{O}:=A_{12} A_{13} A_{14} A_{23} A_{24} A_{34}
$$

form three parallelograms which intersect at the barycenter $C$ of $T$ and split octahedron in eight tetrahedra. The first level of uniform refinement of $T$ is defined as the spliting of $T$ into 12 tetrahedra as shown in Figure 4.4. We note that $T \backslash \mathcal{O}$ consists of four tetrahedra similar with $T$ and that $\mathcal{O}$ is split is in eight tetrahedra which belong to at most four different classes of similarity.

We introduce five parallelism-similarity classes $\mathcal{C}_{0}, \mathcal{C}_{1}, \mathcal{C}_{2}, \mathcal{C}_{3}, \mathcal{C}_{4}$ as follows. Let $\mathcal{C}_{0}$ be the class containing $T_{0}:=A_{1} A_{2} A_{3} A_{4}, \mathcal{C}_{1}$ be the class containing $T_{1}:=$ $C A_{12} A_{13} A_{14}, \mathcal{C}_{2}$ be the class containing $T_{2}:=C A_{12} A_{23} A_{24}, \mathcal{C}_{3}$ be the class containing $T_{3}:=C A_{13} A_{23} A_{34}$ and $\mathcal{C}_{4}$ be the class containing $T_{4}:=C A_{14} A_{24} A_{34}$. We say that a tetrahedron $\tau_{i}$ belongs to $\mathcal{C}_{i}$ if $\tau_{i}$ is similar with $T_{i}$ and each edge of $\tau_{i}$ has the same direction with an edge of $T_{i}$. In other words, $\tau_{i}$ can be obtained from $T_{i}$ by applying dilations, translations or point reflections but not rotations. For example $A_{1} A_{12} A_{13} A_{14} \in \mathcal{C}_{0}$ and $C A_{34} A_{24} A_{23} \in \mathcal{C}_{1}$ since the tetrahedron $C A_{34} A_{24} A_{23}$ is the point reflection of the tetrahedron $T_{1}=C A_{12} A_{13} A_{14}$ with respect to $C$. Thus the first level of refinement produces 12 tetrahedra in five classes of parallelismsimilarity.

To describe the next levels of refinement, we shall use affine coordinates relative to $T$. Namely, the point $P$ is associated the affine coordinates $\left[x_{1}, x_{2}, x_{3}, x_{4}\right]$ if $x_{1}, x_{2}, x_{3}, x_{4}$ are the unique real numbers such that $x_{1}+x_{2}+x_{3}+x_{4}=1$ and 


$$
\begin{gathered}
O P=x_{1} O A_{1}+x_{2} O A_{2}+x_{3} O A_{3}+x_{4} O A_{4} . \text { Then } T=\left\{x_{j} \geq 0\right\} . \text { We have that } \\
A_{1}=[1,0,0,0], \quad A_{2}=[0,1,0,0], \quad A_{3}=[0,0,1,0], \quad A_{4}=[0,0,0,1], \\
A_{12}=[1,1,0,0] / 2, \quad A_{13}=[1,0,1,0] / 2, \quad A_{14}=[1,0,0,1] / 2, \\
A_{23}=[0,1,1,0] / 2, \quad A_{24}=[0,1,0,1] / 2, \quad A_{34}=[0,0,1,1] / 2, \\
\text { and } C=[1,1,1,1] / 4 .
\end{gathered}
$$

Thus, the nodal points associated with the first level of uniform of refinement are points of the form

$$
\left[k_{1}, k_{2}, k_{3}, k_{4}\right] / 4, \quad k_{1}+k_{2}+k_{3}+k_{4}=4,
$$

where $k_{1}, k_{2}, k_{3}, k_{4}$ are non-negative integers which are either all even or $k_{1}=k_{2}=$ $k_{3}=k_{4}=1$ (this last case corresponds to the point $C$ )

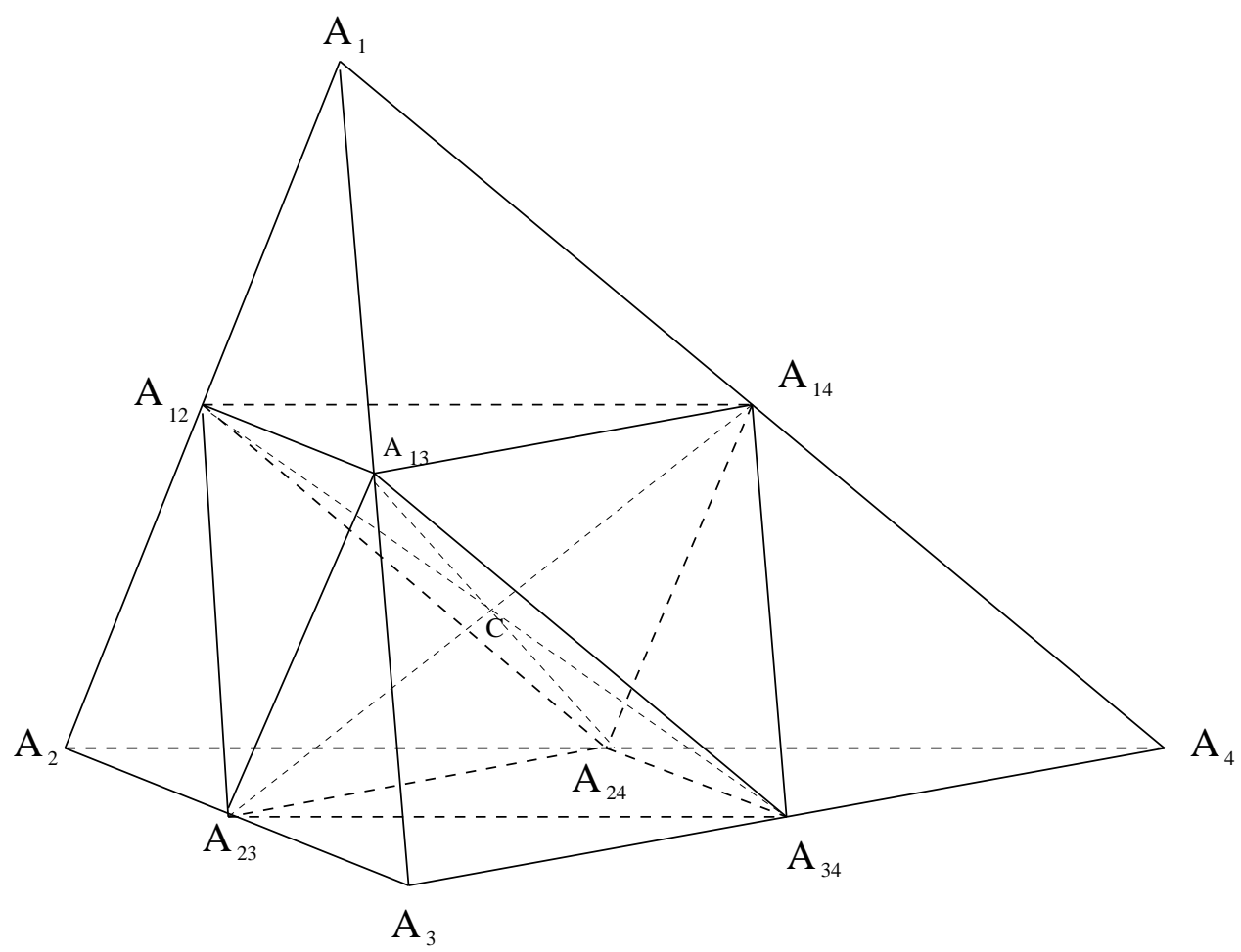

FiguRE 4.4. First level of uniform refinement

We then define the level $n$ of uniform refinement of $T$ to consist of all the region in which $T$ is divided by the planes given in affine coordinates by :

$x_{i}=k 2^{-n}$ and $x_{i}+x_{j}=k 2^{-n}, \quad i, j=1,2,3,4$, and $k \in\left\{0,1,2, \ldots, 2^{n}-1\right\}$.

If we fix $x_{i}=k 2^{-n}$, we see that the traces of the remaining planes on $x_{i}=k 2^{-n}$ are given by $x_{j}=l 2^{-n}$, so the triangle cut by $x_{i}=k 2^{-n}$ from the tetrahedron $T$ is divided into $\left(2^{n}-k\right)^{2}$ congruent triangles. If we ignore the planes $x_{i}+x_{j}=k 2^{-n}$, then $T$ is is decomposed into $2^{n}\left(2^{n}+1\right)\left(2^{n}+2\right) / 6$ tetrahedra similar and with the same orientation as the initial tetrahedron $T$, into $2^{n}\left(2^{n}-1\right)\left(2^{n}-2\right) / 6$ tetrahedra similar but with the opposite orientation as the initial tetrahedron $T$ (all tetrahedra 
are similar by a factor of $2^{-n}$ to $\left.T\right)$, and of $2^{n}\left(2^{n}-1\right)\left(2^{n}+1\right) / 6$ regions similar to $\mathcal{O}$ (by a factor of $2^{1-n}$ ). This is seen by counting the regions between the planes $x_{i}=\left(2^{n}-k\right) 2^{-n}$ and $x_{i}=\left(2^{n}-k-1\right) 2^{-n}$ : then there are $k(k+1) / 2$ same direction thetrahedra, $k(k-1) / 2$ octahedra, and $(k-1)(k-2) /$ opposite direction tetrahedra.

Each region similar to $\mathcal{O}$ will be then divided into eight tetrahedra (again similar to those $\mathcal{O}$ has been divided into at the first level of refinement). This shows that all our tetrahedra belong to the similarity classes $\mathcal{C}_{0}, \ldots, \mathcal{C}_{4}$. This shows that the level $n$ of uniform refinement leads to a decomposition of our given tetrahedron $T$ of type $\mathbf{S}^{4}$ into $\left(5 \cdot 2^{3 n}-2^{n+1}\right) / 3$ tetrahedra. For $n=2$, we thus obtain 104 tetrahedra (for $n=1$, this formula gives 12 tetrahedra, wich is consistent with our previous observations and with the figure 4.4).

It is interesting to mention that the octahedron $\mathcal{O}$ is divided into 56 tetrahedra in the second level of uniform refinement, so a complete picture of the second level of refinement would be useless. See, however, figure 4.5.

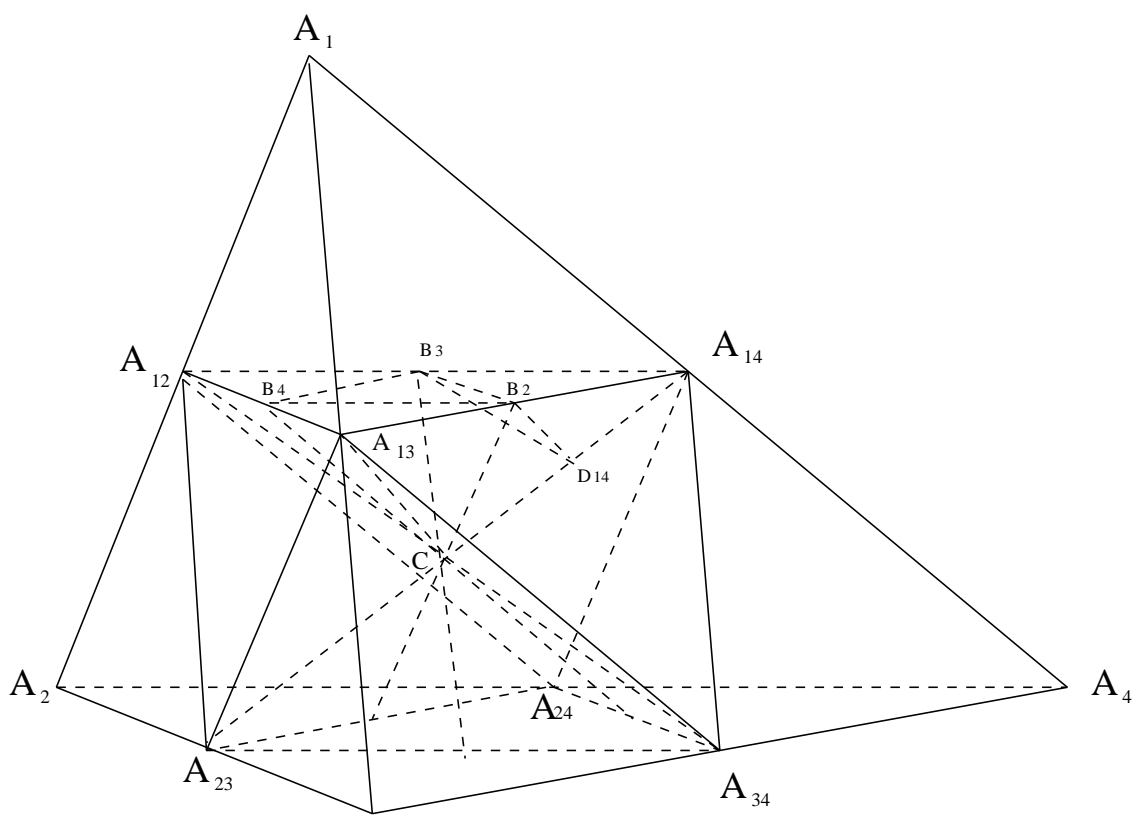

$\mathrm{A}_{3}$

FiguRE 4.5. Refinement of one tetrahedron from $\mathcal{C}_{1}$.

We define the distance between $P\left[x_{1}, x_{2}, x_{3}, x_{4}\right]$ and $Q\left[y_{1}, y_{2}, y_{3}, y_{4}\right]$ by

$$
d(P, Q):=\left|x_{1}-y_{1}\right|+\left|x_{2}-y_{2}\right|+\left|x_{3}-y_{3}\right|+\left|x_{4}-y_{4}\right| .
$$

The nodal points of the second level of uniform refinement are all the old points of the first level refinement together with all mid points of the edges of the first level refinement and can be characterized as the points of the form

$$
\begin{gathered}
{\left[k_{1}, k_{2}, k_{3}, k_{4}\right] / 4, \quad k_{1}+k_{2}+k_{3}+k_{4}=4, \quad \text { and }} \\
{\left[k_{1}, k_{2}, k_{3}, k_{4}\right] / 4+[1,1,1,1] / 8, \quad k_{1}+k_{2}+k_{3}+k_{4}=2,}
\end{gathered}
$$


where $k_{1}, k_{2}, k_{3}, k_{4}$ are non-negative integers. A nodal point will be called even if it is of the first type and odd otherwise. At each level a nodal point will be named even if it is at the intersection of the planes $x_{j}=k 2^{-n}$ and will be called odd otherwise. The odd points are the centers of the octohedra. For example, $C$ is odd in the first level and even in the second level. The faces of the second level of refinement are of two types. The first type consists of triangular faces with all the vertices and mid points (of the second level refinement) being even points. To refine this type, just connect the mid points. The second type has all the vertices and one mid point as even points and the two remaining mid points are odd points. We split this triangular face in four triangles by connecting the even mid point with the opposite vertex and with the other two odd mid points. The refinement of faces induces the refinement of the tetrahedra. The tetrahedra of $\mathcal{C}_{0}$ are refined in the same manner we refined $T$. A tetrahedron from $\mathcal{C}_{1}$ will be refine as shown in Figure 4.5. One can see from Figure 4.5 that a tetrahedron from class $\mathcal{C}_{1}$ is split in seven smaller tetrahedra: one in class $\mathcal{C}_{0}$ three in the same class $\mathcal{C}_{1}$ and one for each of the classes $\mathcal{C}_{2}, \mathcal{C}_{3}$ and $\mathcal{C}_{4}$. The splitting of the rest of the tetrahedra of the first level of refinement from $\mathcal{C}_{2}, \mathcal{C}_{3}, \mathcal{C}_{4}$ is similar.

Note that the sides of all the tetrahedra in the second level of refinement have length $1 / 2$ in the special metric and refinement preserves the number of parallelismsimilarity classes. The refinement process can continue and it can be proved by induction that a new level of refinement will preserve the number of parallelismsimilarity classes and the conformity of the mesh.

Since the strategy presented for refining a tetrahedron is symmetric with respect to the four vertices (or faces) of the tetrahedron the method extends naturally to the case of polyhedral domains which can be split as union of tetrahedra such that any two tetrahedra are disjoint or share only a vertex or an edge or a face.

4.4. Non-uniform refinement. Let $T$ be be a tetrahedron of type VESS or $\mathbf{V S}^{3}$ of some decomposition $\mathcal{T}_{n}$. It will follow by induction that every tetrahedron in the decompositions $\mathcal{T}_{k}, k \geq 0$, is of the type VESS, VS $\mathbf{S}^{3}$, or $\mathbf{S}^{4}$. We begin by dividing first each of the edges and then each of the faces of $T$ as described in Subsection 4.1.

If $T$ is a tetrahedron of type $\mathbf{V S}^{3}$, we divide it the in 12 tetrahedra like in the uniform strategy, but with the edges through the vertex of type $\mathbf{V}$ divided in the ration given by $\kappa$. These tetrahedra belong to $\mathcal{I}_{n+1}$. There will be a tetrahedron of type $\mathbf{V S} \mathbf{S}^{3}$ and 11 tetrahedra of type $\mathbf{S}^{4}$. See Figure 4.6 Then, as explained in the previous subsection, we shall iterate this construction for the tetrahedron of type $\mathbf{V} \mathbf{S}^{3}$, whereas the tetrahedra of type $\mathbf{S}^{4}$ are divided according to the uniform strategy.

If, on the other hand, $T$ is a tetrahedron of type VESS, we divide it into 6 tetrahedra of type $\mathbf{S}^{4}$, one tetrahedron of type $\mathbf{V S}^{3}$, and a prism. The vertex of type $\mathbf{E}$ of $T$ will belong only to the prism. This division is obtained by first dividing it into 12 pieces like in the uniform strategy. The union of the tetrahedron containing the vertex of type $\mathbf{E}$ and of the tetrahedra adjacent to it will form the prism. In particular, each face is divided according to the previously explained strategies for dividing faces of type VES, VSS, and ESS. See Figure 4.7. Our choice to use a prism explain our division strategy for the faces of type VES.

4.5. Semi-uniform refinements and conclusion. Let $\Lambda$ be a prism in the division $\mathcal{T}_{n}$. In particular, $\Lambda$ is then a straight triangular prism with a distinguished 


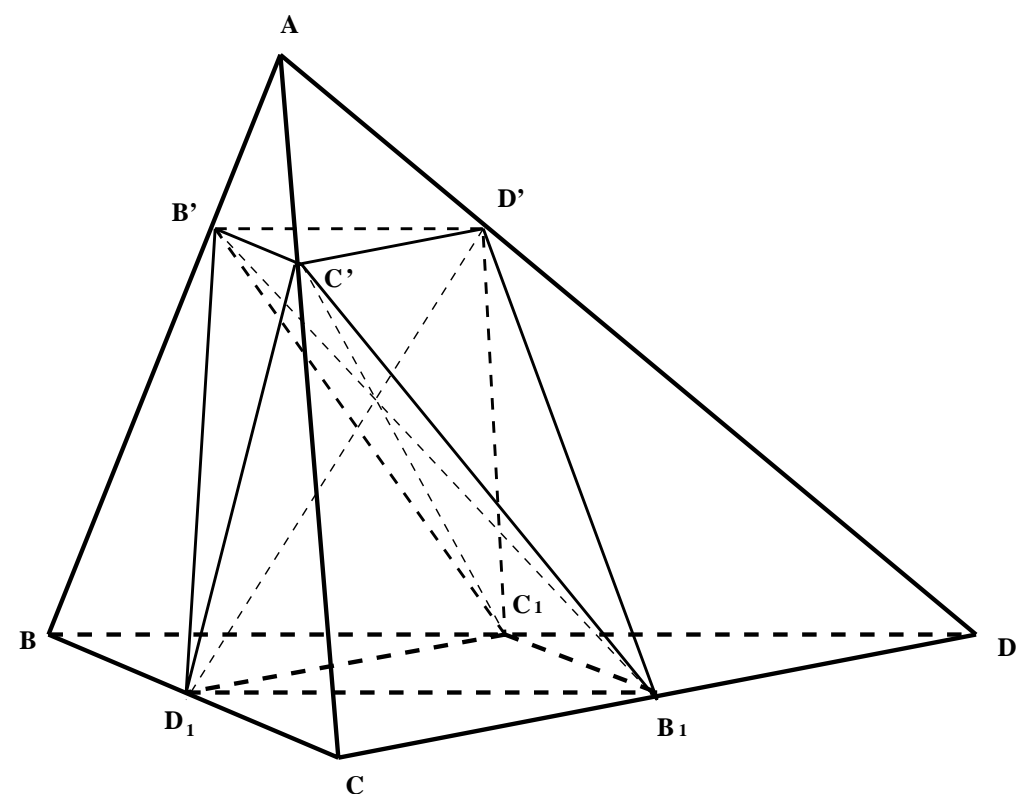

Figure 4.6. A of type $\mathbf{V}, \mathrm{B}, \mathrm{C}, \mathrm{D}$ of type $\mathbf{S}$

diagonal (mark). To obtain $\mathcal{T}_{n+1}$, we divide each parallel edge of $\Lambda$ in 2 equal sides. We also divide each base of $\Lambda$ into four triangles, according to the division strategy of triangles of type ESS, as explained in Subsection 4.1. This yields a decomposition of $\Lambda$ into eight prisms. Each face of $\Lambda$ will be divided according to the prescription of the Subsection 4.1.

Let us now discuss the choice of the marks on the 8 smaller prisms. For $\kappa=1 / 2$, we chose as marks the resulting diagonals parallel to the original mark. For general $\kappa$, we deform this choice from $1 / 2$ to our desired value for $\kappa$. This procedure can be iterated to define the level $k$ of refinement of $\Lambda$, which will yield prisms of $\mathcal{T}_{n+k}$. To obtain the desired tetrahedralization $\mathcal{T}_{n+k}^{\prime}$, we divide each of the resulting $2^{3 k}$ marked prisms in three tetrahedra. See Figure 4.8 for the first level of semi-uniform refinement of a prism. We shall come back to these constructions in Subsection 6.1.

By examining our refinement procedure, we obtain the following.

Theorem 4.1. Let $\mathcal{T}_{n}$ be the sequence of decompositions obtained by applying the procedure outlined in Subsection 4.2 using uniform, semi-uniform, and nonuniform refinements. Then $\mathcal{T}_{n}$ satisfies the Conditions (i-viii) of Subsection 3.2. All tetrahedra of the resulting tetrahedralizations are of the type VESS, $\mathbf{V S}^{3}$, or $\mathbf{S}^{4}$. The region $X:=\Omega \backslash \cup_{P} \mathcal{V}_{P, 1}$ consists of the prisms and the tetrahedra of type $\mathbf{S}^{4}$ of the initial tetrahedralization $\mathcal{T}_{0}^{\prime}$ and of $\mathcal{T}_{1}^{\prime}$.

In particular, we have the following

Corollary 4.2. The mesh $\mathcal{T}_{n}^{\prime}$, when restricted to $\Omega \backslash \cup_{P} \mathcal{V}_{P, 0}$ consists of the level $n$ refinements (uniform and semi-uniform) of the tetrahedra of type $\mathbf{S}^{4}$ of the initial tetrahedralization and of the prisms of the initial decomposition $\mathcal{T}_{0}$. The mesh $\mathcal{T}_{n}^{\prime}$, when restricted to $\cup_{P}\left(\mathcal{V}_{P, 0} \backslash \mathcal{V}_{P, 1}\right)$ consists of the level $n-1$ refinements (uniform 


\section{$\mathbf{A}$}

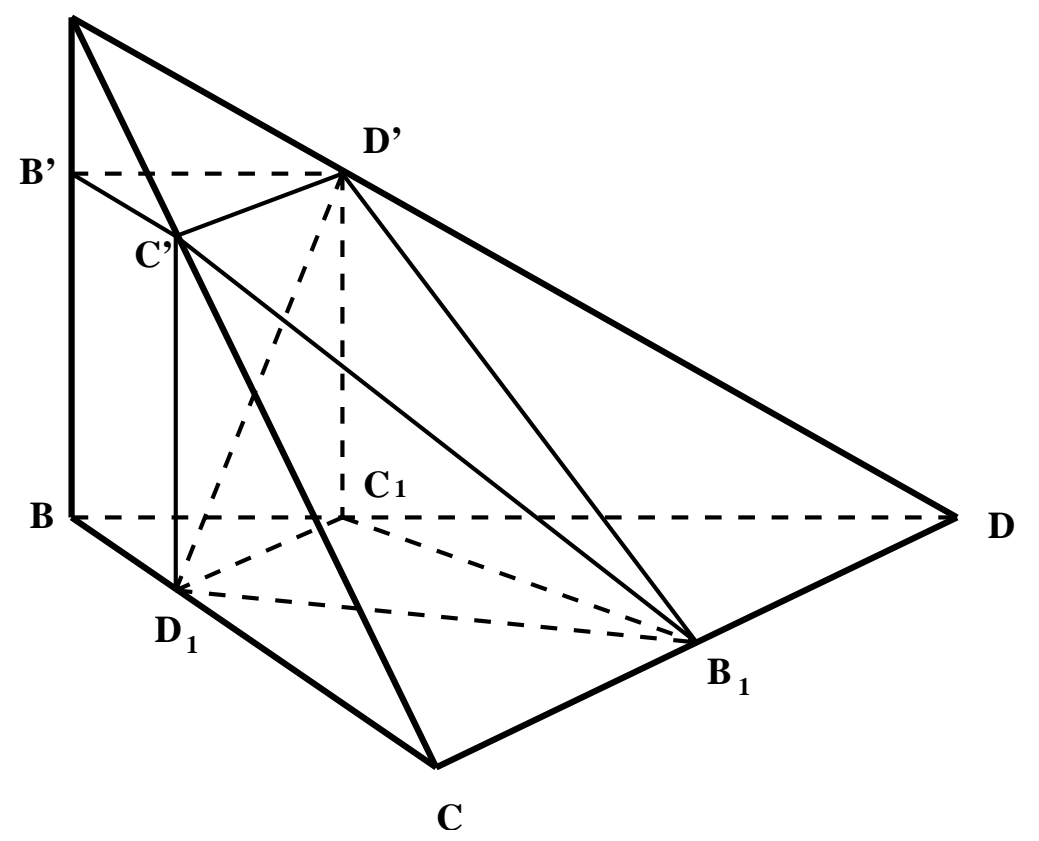

Figure 4.7. A of type $\mathbf{V}, \mathrm{B}$ of type $\mathbf{E}, \mathrm{C}, \mathrm{D}$ of type $\mathbf{S}$ and $D_{1} D^{\prime}=$ mark for the prism $B D_{1} C_{1} D^{\prime} C_{1} B^{\prime}$

and semi-uniform) of the tetrahedra of type $\mathbf{S}^{4}$ and of the prisms obtained by the non-uniform refinement of the tetrahedra in the initial decomposition $\mathcal{T}_{0}$.

Note that no tetrahedron in the initial decomposition is of type $\mathbf{S}^{4}$. (However, the region $\Lambda_{0}$ is divided into tetrahedra of type $\mathbf{S}^{4}$ to yield $\mathcal{T}_{1}^{\prime}$, the initial tetrahedralization.)

\section{INTERPOLATION AND APPROXIMATION ON STANDARD SIMPLICES}

Let $T \subset \mathbb{R}^{3}$ be a tetrahedron with vertices $A, B, C$, and $D$. Let $L_{m}(T) \subset T$ be the set of nodes corresponding to the "linear $m$ simplex" (in the terminology of [16]). In affine coordinates $\left[\lambda_{0}, \lambda_{1}, \lambda_{2}, \lambda_{3}\right] \in \mathbb{R}^{3}, \sum \lambda_{i}=1, \lambda_{i} \geq 0$, we have $L_{m}(T)=$ $\left\{m^{-1}\left[k_{0}, \ldots, k_{3}\right] \in T, k_{j} \in \mathbb{Z}_{+}\right\}$. Thus, for $m=1$, we have $L_{1}(T)=\{A, B, C, D\}$, which corresponds to piecewise linear interpolation.

For $u \in \mathcal{C}(T)$, we shall denote by $I_{T, m}(u)$ the Lagrange interpolant associated to $L_{m}(T)$. It is the unique polynomial of order $m$ such that $I_{T, m}(u)(x)=u(x)$ for all $x \in L_{m}(T)$ [16]. If $T$ and $m$ are clear from the context, we shall also write $u_{I}=I(u)=I_{T, m}(u)$.

Let us consider a prism $A B C A^{\prime} B^{\prime} C^{\prime} \subset \mathbb{R}^{3}$. We assume that $A B C$ and $A^{\prime} B^{\prime} C^{\prime}$ are congruent triangles lying in parallel planes such that $A A^{\prime}, B B^{\prime}$, and $C C^{\prime}$ are perpendicular to the planes $A B C$ and $A^{\prime} B^{\prime} C^{\prime}$. In particular, $A A^{\prime}, B B^{\prime}$, and $C C^{\prime}$ parallel and congruent (i.e., of the same length). A triangular prism with these properties will be called a straight triangular prism. 


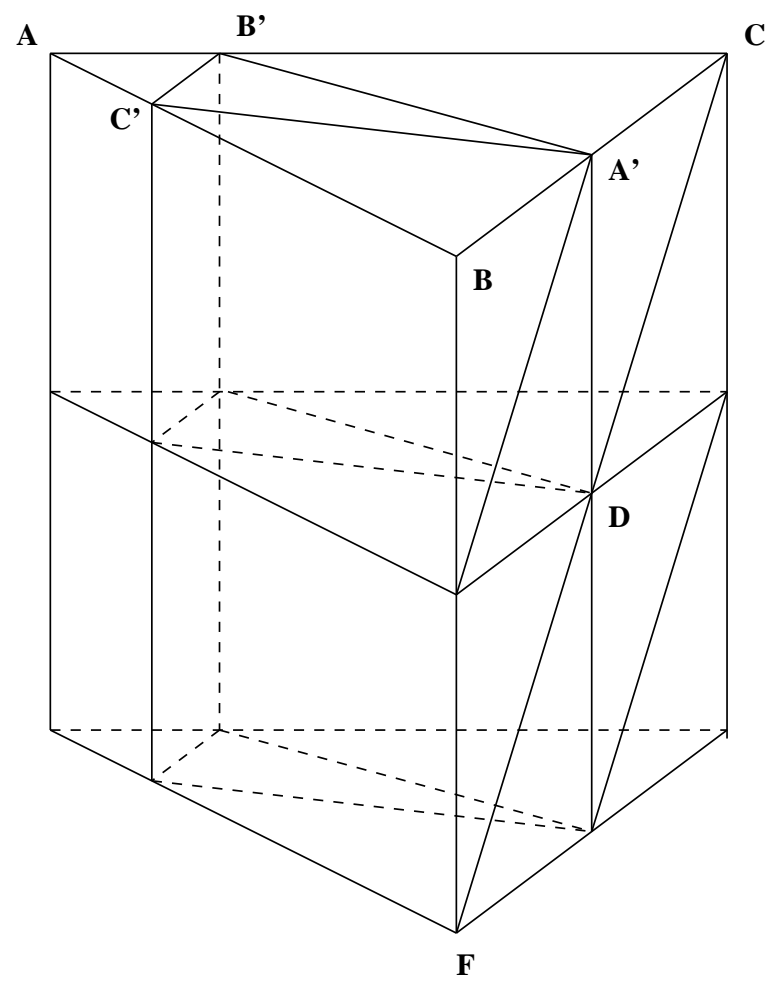

FigURE 4.8. First level of semi-uniform refinement of a prism, $C D=\operatorname{mark}$

We can chose our coordinate system such that $A$ is the origin and $A B C$ lies in the coordinate plane $0 x y$. Then $A^{\prime}$ will be on the $O z$ axis. We shall chose our coordinate system so that the $z$ component of $A^{\prime}$ is positive. We divide $A B C A^{\prime} B^{\prime} C^{\prime}$ into the three tetrahedra $A^{\prime} A B C^{\prime}, A B C C^{\prime}$, and $A^{\prime} B^{\prime} B C^{\prime}$ and let $\hat{\sigma}$ denote any of these tetrahedra. This is the division that we obtain if we consider the prism $A B C A^{\prime} B^{\prime} C^{\prime}$ as being marked by the choice of the diagonal $B C^{\prime}$. This would be a good choice of mark if $A A^{\prime}$ was part of an edge of our polyhedral domain $\Omega$. See Figure 5.1.

The following result should be compared to Lemmas 2.2 and 2.3 in Apel's book [4] (see especially Equations (2.19) and (2.23)).

Theorem 5.1. Let $\omega:=A B C A^{\prime} B^{\prime} C^{\prime}$ be a straight prism divided into three tetrahedra. Let $\hat{\sigma}$ be any of these tetrahedra and $m \geq 2$. Let $u \in \mathcal{C}^{1}(\hat{\sigma})$ and $I(u)=u_{I}$ be interpolant associated to the linear $m$-simplex. Then there exists a constant $C>0$ such that any $u \in H^{m+1}(\omega)$ satisfies

(1) $\partial_{z} u=0$ implies $\partial_{z} I(u)$;

(2) $\left\|\partial_{z}\left(u-u_{I}\right)\right\|_{L^{2}(\hat{\sigma})} \leq \hat{C}\left|\partial_{z} u\right|_{H^{m}(\hat{\sigma})}$;

(3) $\partial_{x} u=0$ and $\partial_{y} u=0$ imply $\partial_{x} I(u)$ and $\partial_{y} I(u)$;

(4) $\left\|\partial_{x}\left(u-u_{I}\right)\right\|_{L^{2}(\hat{\sigma})}+\left\|\partial_{y}\left(u-u_{I}\right)\right\|_{L^{2}(\hat{\sigma})} \leq \hat{C}\left(\left|\partial_{x} u\right|_{H^{m}(\hat{\sigma})}+\left|\partial_{y} u\right|_{H^{m}(\hat{\sigma})}\right)$.

Proof. Let $I_{2}$ be the two dimensional interpolant associated with triangle $A B C$ (the basis of our prism). Let $u \in H^{m+1}(\hat{\sigma}) \subset C^{1}(\hat{\sigma})$, for one of the three reference 


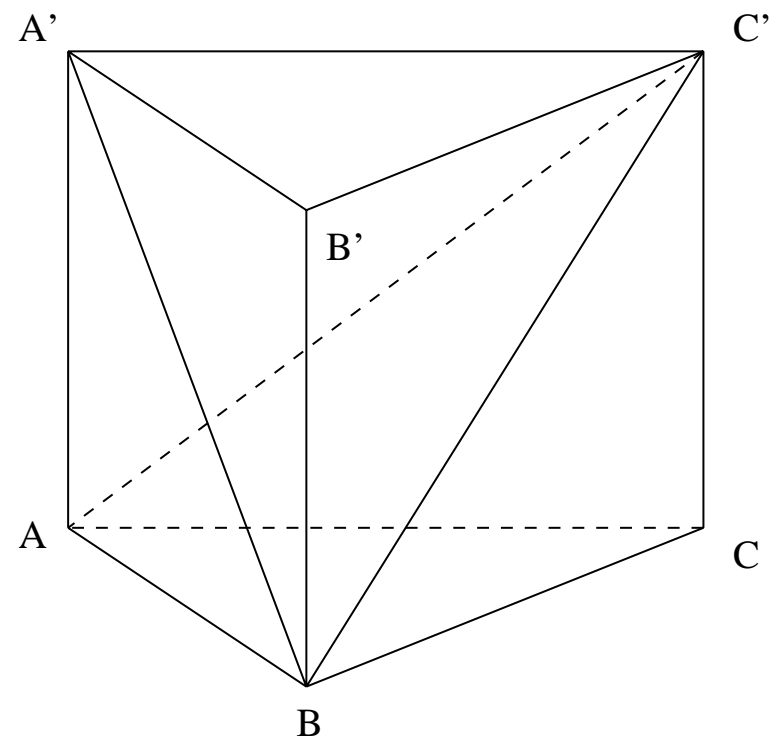

Figure 5.1. Marking a prism: $B C^{\prime}=\operatorname{mark}, A A^{\prime}\left\|B B^{\prime}\right\| C C^{\prime} \perp$ $A B C$ and $A^{\prime} B^{\prime} C^{\prime}$

tetrahedra $\hat{\sigma}$. Assume that $\partial_{z} u=0$. Then $u$ is independent of $z$. We then notice that the projection of $\hat{\sigma}$ onto the $x y$-coordinate plane is the triangle $A B C$ and that the nodes of $\hat{\sigma}$ project onto the nodes of $A B C$. If follows that the function $w(x, y, z):=I_{2}\left(\left.u\right|_{A B C}\right)(x, y)$ is such $w=u$ at the nodes of $\hat{\sigma}$. Therefore $I(u)=w$, which is independent of $z$ as well. This proves Part (1).

Part (3) is proved similarly, but with the triangle $A B C$ replaced with the segment $A A^{\prime}$ of the $O z$ axis.

Assume now (1) and let $v=\partial_{z} u$. Then $\partial_{z}\left(u-u_{I}\right)$ depends only on $v$ in the sense that if $\partial_{z} u=\partial_{z} u_{1}$, then $\partial_{z}\left(u-u_{I}\right)-\partial_{z}\left(u_{1}-u_{1 I}\right)=\partial_{z}\left(\left(u-u_{1}\right)-\left(u-u_{1}\right)_{I}\right)=0$. Define then $F: H^{m}(\hat{\sigma}) \rightarrow L^{2}(\hat{\sigma})$ by $F(v)=\partial_{z}\left(u-u_{I}\right)$, where $u \in H^{m}(\hat{\sigma})$ is any function such that $v=\partial_{z} u$. If $v$ is a polynomial of order $m-1$, we let $u(x, y, z)=\int_{0}^{z} v(x, y, \zeta) d \zeta$, which is a polynomial of degree at most $m$ satisfying $\partial_{z} u=v$. Then $u=u_{I}$, and hence we have $F(v)=0$ for any polynomial $v$ of order $m-1$. Since $F$ is continuous (for $m \geq 2$ ), we have by the Bramble-Hilbert lemma that

$$
\left\|\partial_{z}\left(u-u_{I}\right)\right\|_{L^{2}(\hat{\sigma})}:=\|F(v)\|_{L^{2}(\hat{\sigma})} \leq C|v|_{H^{m}(\hat{\sigma})} .
$$

This proves Part (2).

The proof of Part (4), is similar. Let us consider the map $D u=\left(\partial_{x} u, \partial_{y} y\right)$, with $u \in H^{m+1}(\hat{\sigma})$. Let $V \subset H^{m}(\hat{\sigma}) \oplus H^{m}(\hat{\sigma})$ be the closed subspace of pairs $(f, g)$ such that $\partial_{y} f=\partial_{x} g$. Then $D u \in V$. We define a map $F: V \rightarrow V$ by $F(f, g)=\left(\partial_{x}\left(u-u_{I}\right), \partial_{y}\left(u-u_{I}\right)\right)$, where $u$ is such that $\partial_{x} u=f$ and $\partial_{y} u=g$. By Part (3), this definition is independent of $u$. In fact, we can chose $u(x, y)=$ $\int_{0}^{1}(f(t x, t y) x+g(t x, t y) y) d t=\int_{(0,0)}^{(x, y)}(f d x+g d y)$, which shows that if $f$ and $g$ are polynomials of degree $\leq m-1$, then we can chose $u$ to be a polynomial of degree $\leq m$. Consequently, $F(f, g)=0$ if $f$ and $g$ are polynomials of degree at most $m-1$. 
This shows that

$$
\begin{aligned}
&\left\|\partial_{x}\left(u-u_{I}\right)\right\|_{L^{2}(\hat{\sigma})}^{2}+\left\|\partial_{y}\left(u-u_{I}\right)\right\|_{L^{2}(\hat{\sigma})}^{2}:=\|F(f, g)\|_{L^{2}(\hat{\sigma})}^{2} \\
& \leq C|(f, g)|_{H^{m}(\hat{\sigma})}^{2}:=|f|_{H^{m}(\hat{\sigma})}^{2}+|g|_{H^{m}(\hat{\sigma})}^{2},
\end{aligned}
$$

again by the Bramble-Hilbert lemma. This proves Part (4).

\section{INTERPOLATION ON THIN TETRAHEDRA}

We now use the results of the previous section to establish that the sequence of tetrahedralizations defined in Section 4 satisfies also Condition ix. (All the other conditions required in Subsection 3.2 were already verified in Section 4.

The verification of Condition (ix) essentially reduces to the verification of the analogous statement for each straight triangular prism $\Lambda$ appearing in the initial decomposition $\mathcal{T}_{0}$ of $\Omega$ (we shall give details below). Let then $\Lambda=A B C A^{\prime} B^{\prime} C^{\prime}$ be a straight triangular prism as in Section 5. (Recall from Section 5 that a straight triangular prism is a triangular prism such that $A A^{\prime}, B B^{\prime}$, and $C C^{\prime}$ are perpendicular to the planes of the triangles $A B C$ and $A^{\prime} B^{\prime} C^{\prime}$.) We shall chose our notation so that $A A^{\prime}$ lies on an edge of $\Omega$. Then, recall from Subsection 1.2, that the $\bar{\Lambda}$ intersects the edges of $\Omega$ in $A A^{\prime}$, so no other points of $\bar{\Lambda}$, except those on $A A^{\prime}$, lie on an edge of $\Omega$. Moreover, no vertex of $\Omega$ belongs to $\bar{\Lambda}$.

We can also assume that $A$ is the origin of the coordinate systems and that $A^{\prime}$ is on the positive $O z$ coordinate semi-axis. (An then $A B C$ is in the $x y$ coordinate plane.)

6.1. Prism divisions and tetrahedralizations. We shall denote by $\mathcal{T}_{n}(\Lambda)$ and $\mathcal{T}_{n}^{\prime}(\Lambda)$ the restrictions to $\Lambda$ of the divisions $\mathcal{T}_{n}$ and of the tetrahedralizations $\mathcal{T}_{n}^{\prime}$ of $\Omega$. Recall then that $\mathcal{T}_{n}(\Lambda)$ consists of straight prisms that are obtained from a triangulation $\tau_{n}$ of $A B C$ and the division of the edges $A A^{\prime}, B B^{\prime}$, and $C C^{\prime}$ into $2^{n}$ equal segments. The triangulation $\tau_{n}$ consists of $2^{2 n}$ triangles obtained by induction as explained in Subsection 4.1.

Recall from Subsection 4.1 that each triangle in $\tau_{n}$ is obtained by dividing each triangle in $\tau_{n-1}$ into four other triangles. There are two types of triangles in $\tau_{n-1}$ : type ESS and $\mathbf{S}^{3}$. A triangle of type $\mathbf{S}^{3}$ in the triangulation $\tau_{n-1}$ is divided uniformly, that is, in four equal triangles, by dividing each side in two. A triangle of type ESS in the triangulation $\tau_{n-1}$ is still divided into four tringles, but these triangles will not be equal, unless $\kappa=1 / 2$. Therefore, a triangle of type ESS is divided non-uniformly. This division is obtained by deforming the uniform division so that the points on each side are closer to the singular point and divide that side into the ration $\kappa(1-\kappa)^{-1}$. The resulting non-uniform division is the same as the one considered in [14].

In any case, the resulting triangles in $\tau_{n}$ can be divided into four groups, each similar to one of the triangles of $\tau_{0}$. (The small triangle of $\tau_{0}$ at the vertex of $A B C$ is similar to $A B C$.) More precisely, consider the family of affine maps

$$
\Phi^{\prime}:=\left\{\phi(x, y)=\left(\lambda x+x_{0}, \lambda y+y_{0}\right)\right\}, \text { with } \lambda= \pm \kappa^{j-1} 2^{j-n}, 1 \leq j \leq n .
$$

Then each of the triangles defining the triangulation $\tau_{n}$ is mapped by one of the affine maps $\phi^{-1}, \phi \in \Phi^{\prime}$, to one of the four triangles of the triangulation $\tau_{1}$.

The division $\mathcal{T}_{n}(\Lambda)$ of the prism $\Lambda=A B C A^{\prime} B^{\prime} C^{\prime}$ is then obtained by first dividing $\mathcal{T}_{n}(\Lambda)$ into $2^{2 n}$ straight triangular prisms with bases the triangles in $\tau_{n}$ and 
their counterparts in $A^{\prime} B^{\prime} C^{\prime}$. Then each of these $2^{2 n}$ thin straight triangular prisms is further divided into $2^{n}$ equal straight triangular prisms using planes parallel to the bases.

We also need to explain how the resulting $2^{3 n}$ small prisms are marked. Recall first that $\Lambda$ is marked (that is, one of the diagonals $B C^{\prime}$ or $B^{\prime} C$ opposite to the edge $A A^{\prime}$ was chosen, also recall, that the marks on the prisms that have a face in common have to be compatible in the sense that their marks intersect in one of the common vertices). Assume that the initial mark is $B C^{\prime}$ and that that $n=1$. Then we need to specify 8 marks (or diagonals). When possible, we shall chose the diagonal (or mark) parallel to the initial mark. This is possible for (at least) four prisms. When $\kappa=1 / 2$ (and only then) this is possible for all our eight prisms, with two pairs of prisms sharing one two of these diagonals. For general $\kappa$, we continuously deform from $\kappa=1 / 2$ to our value. This procedure generalizes to define the marks of the prisms of the level $n$ semi-uniform division of $\Lambda$. To obtain the tetrahedralization $\mathcal{T}_{n}^{\prime}(\Lambda)$ of $\Lambda$, we first mark a face of each of the $2^{3 n}$ prisms in $\mathcal{T}_{n}(\Lambda)$ and then we divide each of these prisms in three tetrahedra, as explained in Subsection 3.1.

Let us consider the family of affine maps

$$
\Phi:=\left\{\phi(x, y, z)=\left(\lambda x+x_{0}, \lambda y+y_{0}, 2^{1-n} z+z_{0}\right)\right\}
$$

with $\lambda= \pm \kappa^{j-1} 2^{j-n}$ and $1 \leq j \leq n$ as in the definition of the family $\Phi^{\prime}$ above. Then each of the prisms in $\mathcal{T}_{n}(\Lambda)$ is mapped by some affine map $\phi^{-1}, \phi \in \Phi$, to a prism in $\mathcal{T}_{1}(\Lambda)$. The 12 tetrahedra of $\mathcal{T}_{1}^{\prime}(\Lambda)$ will be called standard simplices. Our affine maps preserve the marks and hence can be used to map each simplex in $\mathcal{T}_{n}^{\prime}(\Lambda)$ to one of the 12 standard simplices and denote them $\hat{\sigma}$.

The following proposition is the crucial step in checking condition (ix).

Lemma 6.1. Let $T$ be a tetrahedron in the level $n$ decomposition $\mathcal{T}_{n}^{\prime}(\Lambda)$. Also, let $u_{I}$ be the interpolant of $u$ associated to the Lagrange $m$-simplex, $m \geq 2$ and $0<\kappa \leq 2^{-m / a}$. Then

$$
\left|u-u_{I}\right|_{H^{1}(T)} \leq C_{0} 2^{-n m}\|u\|_{\mathcal{D}_{a+1}^{m+1}(T)},
$$

for a constant $C_{0}>0$ that depends only on the initial decomposition $\mathcal{T}_{0}$ of $\Omega$, on $m$, and on $\kappa$, but not on $T$ or $n$.

Proof. Let $\phi(x, y, z)=\left(\lambda x+x_{0}, \lambda y+y_{0}, 2^{1-n} z+z_{0}\right)$ be the affine map that sends one of the standard simplexes $\hat{\sigma}$ (i.e., one of the tetrahedra of $\mathcal{T}_{1}^{\prime}(\Lambda)$ ) to $T$ bijectively. Recall that $\lambda= \pm \kappa^{j-1} 2^{j-n}$ and $1 \leq j \leq n$.

We shall write

$$
\hat{u}:=u \circ \phi \in \mathcal{D}_{a+1}^{m+1}(\hat{\sigma})
$$

for any $u \in \mathcal{D}_{a+1}^{m+1}(T)$. It is a standard fact that $\widehat{v}_{I}=(\hat{v})_{I}$. We shall also write $\partial_{1}=\partial_{x}, \partial_{2}=\partial_{y}$, and $\partial_{3}=\partial_{z}$, and $\partial^{\alpha_{\perp}}=\partial_{1}^{\alpha_{1}} \partial_{2}^{\alpha_{2}}$ if $\alpha_{\perp}=\left(\alpha_{1}, \alpha_{2}\right) \in \mathbb{Z}_{+}^{2}$. Let $\delta$ be the Jacobian of $\phi$. 
Let us fix $u \in \mathcal{D}_{a+1}^{m+1}(T)$. Then Theorem 5.1(4) gives, for $\left|\alpha_{\perp}\right|=\alpha_{1}+\alpha_{2}=m-\gamma$,

$$
\begin{aligned}
& \text { (29) } \quad|\lambda|^{2} \delta \sum_{i=1}^{2}\left\|\partial_{i} u-\partial_{i} u_{I}\right\|_{L^{2}(T)}^{2}=|\lambda|^{2} \sum_{i=1}^{2}\left\|\widehat{\partial_{i} u}-\widehat{\partial_{i} u_{I}}\right\|_{L^{2}(\hat{\sigma})}^{2} \\
& =\sum_{i=1}^{2}\left\|\partial_{i} \hat{u}-\partial_{i} \widehat{u_{I}}\right\|_{L^{2}(\hat{\sigma})}^{2}=\sum_{i=1}^{2}\left\|\partial_{i} \hat{u}-\partial_{i}(\hat{u})_{I_{I}}\right\|_{L^{2}(\hat{\sigma})}^{2} \leq C \sum_{i=1}^{2}\left|\partial_{i} \hat{u}\right|_{H^{m}(\hat{\sigma})}^{2} \quad \text { (Thm. 5.1) } \\
& =C \sum_{i=1}^{2} \sum_{\alpha_{\perp}}\left\|\partial^{\alpha_{\perp}} \partial_{z}^{\gamma} \partial_{i} \hat{u}\right\|_{L^{2}(\hat{\sigma})}^{2}=C_{1} \delta \sum_{i=1}^{2} \sum_{\alpha_{\perp}}|\lambda|^{2\left|\alpha_{\perp}\right|+2} 2^{-2 n \gamma}\left\|\partial^{\alpha_{\perp}} \partial_{z}^{\gamma} \partial_{i} u\right\|_{L^{2}(T)}^{2},
\end{aligned}
$$

where the second sum is for all $\alpha_{\perp}=\left(\alpha_{1}, \alpha_{2}\right) \in \mathbb{Z}_{+}^{2}$ such that $\left|\alpha_{\perp}\right|+\gamma=m$, as it will be the case below.

Let $r$ be the distance to the line $A A^{\prime}$ (the supporting an edge of our polyhedral domain $\Omega$ ). Assume first that our tetrahedron $T$ is not adjacent to the edge $A A^{\prime}$. If $\lambda= \pm \kappa^{j-1} 2^{j-n}$, this means that $C_{r} r \geq \kappa^{j}$ on $T$, for a constant that depends only on the initial decomposition $\mathcal{T}_{0}$. Next we use, for $k \geq 1$ and $a \in(0,1]$, the inequality $\|w\|_{L^{2}(T)} \leq C_{r}^{k-a} \kappa^{j(a-k)}\left\|r^{k-a} w\right\|_{L^{2}(T)}$ to obtain, with $C_{1}$ and $\delta$ as in Equation (29) (and $C$ a generic constant that depends only on the initial decomposition $\mathcal{T}_{0}$ ),

$$
\begin{gathered}
C_{1} \delta \sum_{i=1}^{2} \sum_{\alpha_{\perp}}|\lambda|^{2\left|\alpha_{\perp}\right|+2} 2^{-2 n \gamma}\left\|\partial^{\alpha_{\perp}} \partial_{z}^{\gamma} \partial_{i} u\right\|_{L^{2}(T)}^{2} \\
\leq C \delta \sum_{i=1}^{2} \sum_{\left|\alpha_{\perp}\right|>0}|\lambda|^{2\left|\alpha_{\perp}\right|+2} 2^{-2 n \gamma} \kappa^{2 j\left(a-\left|\alpha_{\perp}\right|\right)}\left\|r^{\left|\alpha_{\perp}\right|-a} \partial^{\alpha_{\perp}} \partial_{z}^{\gamma} \partial_{i} u\right\|_{L^{2}(T)}^{2} \\
+C \delta|\lambda|^{2} 2^{-2 n m} \sum_{i=1}^{2}\left\|\partial_{z}^{m} \partial_{i} u\right\|_{L^{2}(T)}^{2} \\
\leq C \delta|\lambda|^{2} \kappa^{-2 m} 2^{-2 n m}\left(\sum_{\left|\alpha_{\perp}\right| \geq 2}\left\|r^{\left|\alpha_{\perp}\right|-a-1} \partial^{\alpha_{\perp}} \partial_{z}^{\gamma} u\right\|_{L^{2}(T)}^{2}+\sum_{i=1}^{2}\left\|\partial_{z}^{m} \partial_{i} u\right\|_{L^{2}(T)}^{2}\right)
\end{gathered}
$$

Combining the Equations (29) and (30), we obtain

$$
\begin{aligned}
& \sum_{i=1}^{2}\left\|\partial_{i} u-\partial_{i} u_{I}\right\|_{L^{2}(T)}^{2} \\
& \quad \leq C_{0} 2^{-2 n m}\left(\sum_{\left|\alpha_{\perp}\right| \geq 2}\left\|r^{\left|\alpha_{\perp}\right|-a-1} \partial^{\alpha_{\perp}} \partial_{z}^{\gamma} u\right\|_{L^{2}(T)}^{2}+\sum_{i=1}^{2}\left\|\partial_{z}^{m} \partial_{i} u\right\|_{L^{2}(T)}^{2}\right)
\end{aligned}
$$

with $C_{0}$ depending on $\kappa$, on $m$, and on the initial decomposition $\mathcal{T}_{0}$, but not on $n$ or $T$ (as long as $\bar{T} \cap A A^{\prime}=\emptyset$ ).

We now treat the $\partial_{z} u$ term. We continue to assume that $T$ is not adjacent to the edge $A A^{\prime}$. We proceed similarly, but using Theorem 5.1(2) instead of Theorem 
5.1(4). We continue to denote $\left|\alpha_{\perp}\right|=\alpha_{1}+\alpha_{2}=m-\gamma$. Then

$$
\begin{gathered}
(32) 2^{-2(n-1)} \delta\left\|\partial_{z} u-\partial_{z} u_{I}\right\|_{L^{2}(T)}^{2}=2^{-2(n-1)}\left\|\widehat{\partial_{z} u}-\widehat{\partial_{z} u_{I}}\right\|_{L^{2}(\hat{\sigma})}^{2} \\
=\left\|\partial_{z} \hat{u}-\partial_{z} \widehat{u_{I}}\right\|_{L^{2}(\hat{\sigma})}^{2}=\left\|\partial_{z} \hat{u}-\partial_{z}(\hat{u})_{I}\right\|_{L^{2}(\hat{\sigma})}^{2} \leq C\left|\partial_{z} \hat{u}\right|_{H^{m}(\hat{\sigma})}^{2}(\mathrm{Thm} .5 .1) \\
=C \sum_{\alpha_{\perp}}\left\|\partial^{\alpha_{\perp}} \partial_{z}^{\gamma+1} \hat{u}\right\|_{L^{2}(\hat{\sigma})}^{2}=C_{1} \delta \sum_{\alpha_{\perp}}|\lambda|^{2\left|\alpha_{\perp}\right|} 2^{-2(n-1)(\gamma+1)}\left\|\partial^{\alpha_{\perp}} \partial_{z}^{\gamma+1} u\right\|_{L^{2}(T)}^{2} \\
\leq C \delta\left(\sum_{\left|\alpha_{\perp}\right| \geq 2}|\lambda|^{2\left|\alpha_{\perp}\right|} 2^{-2(n-1)(\gamma+1)} \kappa^{2 j\left(a+1-\left|\alpha_{\perp}\right|\right)}\left\|r^{\left|\alpha_{\perp}\right|-a-1} \partial^{\alpha_{\perp}} \partial_{z}^{\gamma+1} u\right\|_{L^{2}(T)}^{2}\right. \\
\left.+|\lambda|^{2} 2^{-2(n-1) m} \sum_{i=1}^{2}\left\|\partial_{i} \partial_{z}^{m} u\right\|_{L^{2}(T)}^{2}+2^{-2(n-1)(m+1)}\left\|\partial_{z}^{m+1} u\right\|_{L^{2}(T)}^{2}\right) \\
\leq C \delta \kappa^{-2 m} 2^{-2(n-1)} 2^{-2 n m}\left(\sum_{\left|\alpha_{\perp}\right| \geq 2}\left\|r^{\left|\alpha_{\perp}\right|-a-1} \partial^{\alpha_{\perp}} \partial_{z}^{\gamma+1} u\right\|_{L^{2}(T)}^{2}\right. \\
\left.\quad+\sum_{i=1}^{2}\left\|\partial_{i} \partial_{z}^{m} u\right\|_{L^{2}(T)}^{2}+\left\|\partial_{z}^{m+1} u\right\|_{L^{2}(T)}^{2}\right) .
\end{gathered}
$$

Next, we include the factor $\kappa^{-m}$ into the constant $C$, which will be then denoted $C_{0}$ and will hence depend on $\kappa$. Then we multiply the inequality (32) with $2^{2(n-1)} \delta^{-1}$ and add with the inequality of Equation (31), to obtain (for the last inequality we also use Lemma 1.5)

$$
\begin{aligned}
\left|u-u_{I}\right|_{H^{1}(T)}^{2} & :=\sum_{i=1}^{3}\left\|\partial_{i} u-\partial_{i} u_{I}\right\|_{L^{2}(T)}^{2} \\
\leq & C_{0} 2^{-2 n m} \sum_{i=1}^{2}\left(\sum_{\left|\alpha_{\perp}\right| \geq 2}\left\|r^{\left|\alpha_{\perp}\right|-a-1} \partial^{\alpha \perp} \partial_{z}^{\gamma+1} u\right\|_{L^{2}(T)}^{2}\right. \\
& \left.+\sum_{i=1}^{2}\left\|\partial_{i} \partial_{z}^{m} u\right\|_{L^{2}(T)}^{2}+\left\|\partial_{z}^{m+1} u\right\|_{L^{2}(T)}^{2}\right) \leq C_{0} 2^{-2 n m}\|u\|_{\mathcal{D}_{a+1}^{m+1}(T)}^{2}
\end{aligned}
$$

for a possibly larger constant $C_{0}$ (still independent of $n$ and $T$ ). This proves the desired result as long as $T$ is not adjacent to $A A^{\prime}$.

Assume now that $T$ is adjacent to $A A^{\prime}$. Then $\lambda=\kappa^{n-1}$, so $j=n$. We shall proceed as in the proof of Theorem 3.2, more precisely, we shall used the idea used in the proof of Equation (24).

Let $\chi$ be a smooth function on $\Omega$ such that $\chi=0$ in a neighborhood of the edges and such that $\chi=1$ at all nodal points of $\mathcal{T}_{0}^{\prime}$ not on the edges. Let $u \in \mathcal{D}_{a+1}^{m+1}(T)$. Define $v \in \mathcal{D}_{a+1}^{m+1}(T)$ by $\hat{v}=\chi \hat{u}$. The we still have $C_{r} r \geq \kappa^{j}=\kappa^{n}$, with $C_{r}$ depending only on the initial decomposition $\mathcal{T}_{0}$. Exactly the same proof as above then extends applies to $v$ in place of $u$ to show that

$$
\left|v-v_{I}\right|_{H^{1}(T)} \leq C_{0} 2^{-n m}\|v\|_{\mathcal{D}_{a+1}^{m+1}(T)} .
$$

We again notice that $v_{I}=u_{I}$ (since $u$ and $v$ coincide at the node points). Let $\chi_{0}$ be such that $\hat{\chi}_{0}=\chi$. So $v=\chi_{0} u$. We can assume that the restriction of $\chi_{0}$ to our prism $\Lambda$ is independent of $z$. Then we obtain $\left\|\chi_{0}\right\|_{m+1, \infty}=\|\chi\|_{m+1, \infty}$, and hence 
Corollary 1.6 gives

$$
\|v\|_{\mathcal{D}_{a+1}^{k+1}(T)}=\left\|\chi_{0} u\right\|_{\mathcal{D}_{a+1}^{k+1}(T)} \leq C_{0}\|u\|_{\mathcal{D}_{a+1}^{k+1}(T)}, \quad 0 \leq k \leq m
$$

with $C_{0}$ depending only on the initial decomposition $\mathcal{T}_{0}$. Taking also into account that $\kappa^{n a} \leq 2^{-n m}$, we obtain, using also Equation (16),

$$
\begin{array}{r}
\left|u-u_{I}\right|_{H^{1}(T)} \leq|u-v|_{H^{1}(T)}+\left|v-v_{I}\right|_{H^{1}(T)} \leq C_{0}\left(|u|_{H^{1}(T)}+2^{-n m}\|v\|_{\mathcal{D}_{a+1}^{m+1}(T)}^{2}\right) \\
\leq C_{0}\left(\kappa^{n a}\left|r^{-a} u\right|_{H^{1}(T)}+2^{-n m}\|v\|_{\mathcal{D}_{a+1}^{m+1}(T)}\right) \leq C_{0} 2^{-n m}\left(\|u\|_{\mathcal{D}_{a+1}^{1}(T)}+\|u\|_{\mathcal{D}_{a+1}^{m+1}(T)}\right) \\
\leq C_{0} 2^{-n m}\left(\|u\|_{\mathcal{D}_{a+1}^{m+1}(T)}\right) .
\end{array}
$$

We are ready now to prove one of our main results, stating that Condition (ix) is satisfied for $m \geq 2$ for our sequence of thetrahedralizations.

Theorem 6.2. Let $\mathcal{T}_{n}$ be the sequence of decompositions obtained by applying the procedure outlined in Subsection 4.2 using uniform, semi-uniform, and non-uniform refinements. Let $X:=\Omega \backslash \cup_{P} \mathcal{V}_{P, 1}, m \geq 2$, and $\kappa \leq 2^{-m / a}$. Denote by $u_{I, n}$ the interpolant associated to $\mathcal{T}_{n}^{\prime}$. Then

$$
\left|u-u_{I, n}\right|_{H^{1}(X)} \leq C_{0} 2^{-n m}\|u\|_{\mathcal{D}_{a+1}^{m+1}(X)},
$$

for a constant $C_{0}$ that depends only on the initial tetrahedralization $\mathcal{T}_{0}^{\prime}$, on $m$, and on $\kappa$, but not on $n$ or $u$.

Proof. We now turn to the proof of Condition (ix). Let $X:=\Omega \backslash \cup_{P} \mathcal{V}_{P, 1}$, (as in Condition ix). Recall from Corollary 4.2, that $X$ consists of a union of regions $\Lambda$ that are either marked prisms and or tetrahedra of type $\mathbf{S}^{4}$. To these region we apply either a level $n$ or $n-1$ of uniform or semi-uniform refinement. Let $u_{I, n}$ be the interpolant associated to $\mathcal{T}_{n}^{\prime}$ (which is a mesh on $\Lambda$ ) on each of these regions $\Lambda$. If $\Lambda \subset X:=\Omega \backslash \cup_{P} \mathcal{V}_{P, 1}$ is a prism, then adding the inequalities of Lemma 6.1 for all tetrahedra $T$, we obtain

$$
\left|u-u_{I}\right|_{H^{1}(\Lambda)} \leq C_{0} 2^{-n m}\|u\|_{\mathcal{D}_{a+1}^{m+1}(\Lambda)},
$$

with $C_{0}$ depending only on $\mathcal{T}_{0}, m$, and $\kappa$ (but not on $n$ ).

Equation (36) holds also for $\Lambda$ a tetrahedron of type $\mathbf{S}^{4}$, since all thetrahedra obtained by uniformly dividing $\Lambda$ are similar to a fixed number of tetrahedra and all edges are of the order $2^{-n}$.

Adding all equations (36) for the regions $\Lambda$ comprising $X$, we obtain the desired inequality.

\section{Conclusion}

We summarize here our main results. Let $\mathcal{T}_{0}$ be the initial division of our polyhedral domains in straight triangular prisms, tetrahedra of types VESS and VS ${ }^{3}$ (thus having a vertex in common with $\Omega$ ), and an interior region $\Lambda_{0}$, as in Subsection 1.2. We mark the prisms of $\mathcal{T}_{0}$. The marks will allow us to tetrahedralize $\Omega$ by dividing each prisms in three tetrahedra as determined by the mark and then by tetrahedralizing $\Lambda_{0}$ without introducing additional edges on the boundary of $\Lambda$ (but allowing additional internal edges and vertices). We then apply uniform, semi-uniform, and non-uniform refinements to obtain the divisions $\mathcal{T}_{n}$ of $\Omega$ into 
marked prisms and tetrahedra, as explained in Subsection 4.2. The meshes $\mathcal{T}_{n}$ are obtained by dividing each prism into three tetrahedra as determined by the mark (see as in Figure 5.1). Then the sequences $\mathcal{T}_{n}$ and $\mathcal{T}_{n}^{\prime}$ satisfy the conditions (i-viii) of Subsection 3.2, Theorem 4.1. For $m \geq 2$, the sequence of decomposition $\mathcal{T}_{n}$ and the sequence of meshes $\mathcal{T}_{n}^{\prime}$ satisfy also Condition (ix) of Subsection 3.2, by Theorem 6.2 .

Let $S_{n}$ be the Finite Element spaces of continuous, piecewise polynomials of degree at most $m$ on our sequence of meshes $\mathcal{T}_{n}^{\prime}$ and $u_{n} \in S_{n}$ the discrete (Finite Element) solution of the Poisson problem (1) (recall that this main boundary value problem is $-\Delta u=f, u=0$ at the boundary). Theorem 2.2 then guarantees that there exists $a>0$, depending only on $\Omega$, and a constant $C>0$, depending on $a$ and $\Omega$, such that $\|u\|_{\mathcal{D}_{a+1}^{m+1}(\Omega)} \leq C\|f\|_{H^{m-1}(\Omega)}$. Combining with Theorem 3.3, we obtain our main result:

Theorem 7.1. Let $S_{n}$ be the Finite Element spaces of continuous, piecewise polynomials of degree $m \geq 2$ associated to a tetrahedralization $\mathcal{T}_{n}^{\prime}$ of $\Omega$. Let $a>0$ be as in Theorem 2.2 and $\kappa \leq 2^{-m / a}$. Then there exists $C_{\kappa}>0$ such that

$$
\left|u-u_{n}\right|_{H^{1}(\Omega)} \leq C_{\kappa} \operatorname{dim}\left(S_{n}\right)^{-m / 3}\|f\|_{H^{m-1}(\Omega)},
$$

for all $f \in H^{m-1}(\Omega)$ and all $n \in \mathbb{N}$. Moreover, $\operatorname{dim}\left(S_{n}\right) \sim 2^{3 n}$.

By $\operatorname{dim}\left(S_{n}\right) \sim 2^{3 n}$, we mean that $\operatorname{dim}\left(S_{n}\right) 2^{-3 n} \in\left[c^{-1}, c\right]$ for some $c>0$ independent of $n$.

The interpolation results of this paper hold only for $m \geq 2$, because of this, the final results are proved only for $m \geq 2$ (it is possible that it remain true for $m=1$, but the proof would require a different type of interpolant).

\section{Appendix A. Notation}

The main notation is recalled here, for the benefit of the reader:

By $\Omega$ we denote a fixed bounded polyhedral domain. The spaces $\mathcal{K}_{a}^{m}(\Omega)$ and $\mathcal{D}_{a}^{m}(\Omega)$ were introduced in Section 1.

$\mathcal{T}_{n}$ is a sequence of decompositions of $\Omega$ and $\mathcal{T}_{n}^{\prime}$ is a sequence of meshes obtained from $\mathcal{T}_{n}$ by dividing the prisms into three tetrahedra as determined by the mark, for $n \geq 1$. For $n=0$, we also need to divide the region $\Lambda_{0}$ into tetrahedra without introducing additional edges on the boundary (except the marks).

The space $S_{n}$ is the FEM space of continuous functions on $\Omega$ that coincide with a polynomial of degree $m$ on each of the tetrahedra comprising $\mathcal{T}_{n}^{\prime} . u_{n} \in S_{n}$ denotes the Finite Element approximation of the Poisson problem (1).

\section{REFERENCES}

[1] S. Agmon. Lectures on elliptic boundary value problems. Prepared for publication by B. Frank Jones, Jr. with the assistance of George W. Batten, Jr. Van Nostrand Mathematical Studies, No. 2. D. Van Nostrand Co., Inc., Princeton, N.J.-Toronto-London, 1965.

[2] S. Agmon, A. Douglis, and L. Nirenberg. Estimates near the boundary for solutions of elliptic partial differential equations satisfying general boundary conditions. I. Comm. Pure Appl. Math., 12:623-727, 1959.

[3] B. Ammann and V. Nistor. Weighted sobolev spaces and regularity for polyhedral domains. preprint.

[4] T. Apel. Anisotropic finite elements: local estimates and applications. Advances in Numerical Mathematics. B. G. Teubner, Stuttgart, 1999. 
[5] T. Apel and S. Nicaise. The finite element method with anisotropic mesh grading for elliptic problems in domains with corners and edges. Math. Methods Appl. Sci., 21(6):519-549, 1998.

[6] T. Apel, A.-M. Sändig, and J. Whiteman. Graded mesh refinement and error estimates for finite element solutions of elliptic boundary value problems in non-smooth domains. Math. Methods Appl. Sci., 19(1):63-85, 1996.

[7] D. Arnold and R. Falk. Well-posedness of the fundamental boundary value problems for constrained anisotropic elastic materials. Arch. Rational Mech. Anal., 98(2):143-165, 1987.

[8] I. Babuška. Finite element method for domains with corners. Computing (Arch. Elektron. Rechnen), 6:264-273, 1970.

[9] I. Babuška and A. K. Aziz. Survey lectures on the mathematical foundations of the finite element method. In The mathematical foundations of the finite element method with applications to partial differential equations (Proc. Sympos., Univ. Maryland, Baltimore, Md., 1972), pages 1-359. Academic Press, New York, 1972. With the collaboration of G. Fix and R. B. Kellogg.

[10] I. Babuška, R. B. Kellogg, and J. Pitkäranta. Direct and inverse error estimates for finite elements with mesh refinements. Numer. Math., 33(4):447-471, 1979.

[11] C. Bacuta, V. Nistor, and L. Zikatanov. Improving the rate of convergence of high-order finite elements on polyhedra. I. A priori estimates. Numer. Funct. Anal. Optim., 26(6):613-639, 2005.

[12] S. Brenner and R. Scott. The mathematical theory of finite element methods, volume 15 of Texts in Applied Mathematics. Springer-Verlag, New York, second edition, 2002.

[13] C. Băcuţă, V. Nistor, and L. Zikatanov. Boundary value problems and regularity on polyhedral domains. IMA preprint \#1984, August 2004.

[14] C. Băcuţă, V. Nistor, and L. Zikatanov. Improving the rate of convergence of 'high order finite elements' on polygons and domains with cusps. Numerische Matemathik, 100:165-184, 2005.

[15] A. Buffa, M. Costabel, and M. Dauge. Anisotropic regularity results for Laplace and Maxwell operators in a polyhedron. C. R. Math. Acad. Sci. Paris, 336(7):565-570, 2003.

[16] P. Ciarlet. Basic error estimates for elliptic problems. In Handbook of numerical analysis, Vol. II, Handb. Numer. Anal., II, pages 17-352. North-Holland, Amsterdam, 1991.

[17] L.C. Evans. Partial differential equations, volume 19 of Graduate Studies in Mathematics. American Mathematical Society, Providence, RI, 1998.

[18] G. Folland. Introduction to partial differential equations. Princeton University Press, Princeton, NJ, second edition, 1995.

[19] R. B. Kellogg and J. E. Osborn. A regularity result for the Stokes problem in a convex polygon. J. Functional Analysis, 21(4):397-431, 1976.

[20] V. A. Kondrat'ev. Boundary value problems for elliptic equations in domains with conical or angular points. Transl. Moscow Math. Soc., 16:227-313, 1967.

[21] V. Kozlov, V. Maz'ya, and J. Rossmann. Spectral problems associated with corner singularities of solutions to elliptic equations, volume 85 of Mathematical Surveys and Monographs. American Mathematical Society, Providence, RI, 2001.

[22] J. Lubuma and S. Nicaise. Dirichlet problems in polyhedral domains. II. Approximation by FEM and BEM. J. Comput. Appl. Math., 61(1):13-27, 1995.

[23] V. Maz'ya and J. Roßmann. Weighted $L_{p}$ estimates of solutions to boundary value problems for second order elliptic systems in polyhedral domains. ZAMM Z. Angew. Math. Mech., 83(7):435-467, 2003.

[24] A. Mazzucato and Nistor V. Well posedness and regularity for anisotropic elasticity. work in progress.

[25] M. Mitrea and V. Nistor. Boundary layer potentials on manifolds with cylindrical ends. ESI Preprint.

[26] M. Mitrea and V. Nistor. A note on boundary value problems on manifolds with cylindrical ends. In Aspects of boundary problems in analysis and geometry, volume 151 of Oper. Theory Adv. Appl., pages 472-494. Birkhäuser, Basel, 2004.

[27] S. Nazarov and B. Plamenevsky. Elliptic problems in domains with piecewise smooth boundaries, volume 13 of de Gruyter Expositions in Mathematics. Walter de Gruyter \& Co., Berlin, 1994.

[28] L. A. Oganesjan and L. A. Ruhovec. Variatsionno-raznostnye metody resheniya ellipticheskikh uravnenii. Akad. Nauk Armyan. SSR, Erevan, 1979. 
[29] G. Raugel. Résolution numérique par une méthode d'éléments finis du problème de Dirichlet pour le laplacien dans un polygone. C. R. Acad. Sci. Paris Sér. A-B, 286(18):A791-A794, 1978.

[30] M. Taylor. Partial differential equations I, Basic theory, volume 115 of Applied Mathematical Sciences. Springer-Verlag, New York, 1995.

[31] L. Wahlbin. On the sharpness of certain local estimates for $\stackrel{\circ}{H}^{1}$ projections into finite element spaces: influence of a re-entrant corner. Math. Comp., 42(165):1-8, 1984.

E-mail address: bacuta@math.udel.edu

University of Delaware, Mathematical Sciences, Newark, DE 19716

E-mail address: nistor@math.psu.edu

E-mail address: 1tz@math.psu.edu

Pennsylvania State University, Math. Dept., University Park, PA 16802 